TRANSACTIONS OF THE

AMERICAN MATHEMATICAL SOCIETY

Volume 364, Number 8, August 2012, Pages 4011-4039

S 0002-9947(2012)05404-3

Article electronically published on March 26, 2012

\title{
FORCING, GAMES AND FAMILIES OF CLOSED SETS
}

\author{
MARCIN SABOK
}

\begin{abstract}
We study forcing properties of $\sigma$-ideals generated by closed sets. We show that if a $\sigma$-ideal is $\boldsymbol{\Pi}_{1}^{1}$ on $\boldsymbol{\Sigma}_{1}^{1}$ and generated by closed sets, then it is generated by closed sets in all forcing extensions. This implies that the countable-support iteration of forcings associated with such $\sigma$-ideals is proper. We use it to prove an infinite-dimensional version of the Solecki theorem about inscribing positive $\mathbf{G}_{\delta}$ sets into positive analytic sets.

We also propose a new, game-theoretic, approach to the idealized forcing, in terms of fusion games. We provide a tree representation of such forcings, which generalizes the classical approach to Sacks and Miller forcing.

Among the examples, we investigate the $\sigma$-ideal $\mathcal{E}$ generated by closed null sets and $\sigma$-ideals connected with not piecewise continuous functions. For the first one we show that the associated forcing extensions are of minimal degree. For the second one we show that the associated forcing notion is equivalent to Miller forcing.
\end{abstract}

\section{INTRODUCTION}

Idealized forcing is a technique of applying forcing to descriptive set theory. It was systematically investigated by Zapletal in 21] and 22. If $\mathcal{I}$ is a $\sigma$-ideal on a Polish space $X$, we consider the associated forcing notion $\mathbb{P}_{\mathcal{I}}=\operatorname{Bor}(X) / \mathcal{I}$ (or an equivalent forcing, $\operatorname{Bor}(X) \backslash \mathcal{I}$ ordered by inclusion). Among the well-known examples are Sacks forcing and Miller forcing. The former is associated to the $\sigma$ ideal of countable subsets of the Cantor space (or any other Polish space), and the latter is associated to the $\sigma$-ideal of $\mathbf{K}_{\sigma}$ subsets of the Baire space (i.e. the $\sigma$-ideal generated by compact subsets of $\omega^{\omega}$ ).

Zapletal 22 investigated forcings arising from $\sigma$-ideals generated by closed sets and proved [22, Theorem 4.1.2] that if $\mathcal{I}$ is a $\sigma$-ideal on a Polish space $X$ generated by closed sets, then the forcing $\mathbb{P}_{\mathcal{I}}$ is proper and has continuous reading of names in the topology of $X$.

Definability of a $\sigma$-ideal usually relies on the property called $\Pi_{1}^{1}$ on $\boldsymbol{\Sigma}_{1}^{1}$ (cf. 9, Definition 35.9]), which says that for any analytic set $A \subseteq X^{2}$, the set $\{x \in X$ : $\left.A_{x} \in \mathcal{I}\right\}$ is coanalytic. By classical results of Mazurkiewicz [9, Theorem 29.19] and Arsenin-Kungui [9, Theorem 18.18], both the $\sigma$-ideal of countable subsets of $2^{\omega}$ and the $\sigma$-ideal $\mathbf{K}_{\sigma}$ on $\omega^{\omega}$ are $\boldsymbol{\Pi}_{1}^{1}$ on $\boldsymbol{\Sigma}_{1}^{1}$. It is well known (see [9, Theorem 35.38]) that if $\mathcal{K}$ is a hereditary (i.e. closed under taking subsets) and coanalytic (in the sense of the Effros space) family of closed subsets of $X$, then the $\sigma$-ideal generated by $\mathcal{K}$ is $\Pi_{1}^{1}$ on $\Sigma_{1}^{1}$.

Received by the editors October 15, 2009 and, in revised form, June 18, 2010.

2010 Mathematics Subject Classification. Primary 03E15, 28A05, 54H05.

Key words and phrases. Proper forcing, $\sigma$-ideals.

This research was supported by MNiSW grant N 201361836.

(C)2012 American Mathematical Society 
Note that if $\mathcal{I}$ is a $\boldsymbol{\Pi}_{1}^{1}$ on $\boldsymbol{\Sigma}_{1}^{1} \sigma$-ideal, then it makes sense to define $\mathcal{I}^{W}$ in any model $W$ of ZFC (containing the parameters of the definition) as the family of analytic sets satisfying the $\boldsymbol{\Pi}_{1}^{1}$ definition of $\mathcal{I}$. We prove the following result.

Theorem 1.1. Let $\mathcal{I}$ be $a \Pi_{1}^{1}$ on $\boldsymbol{\Sigma}_{1}^{1} \sigma$-ideal. If $\mathcal{I}$ is generated by closed sets in $V$, then $\mathcal{I}^{W}$ is generated by closed sets in all forcing extensions $V \subseteq W$.

Notice that, as a corollary, we get that if $\mathcal{I}$ is $\boldsymbol{\Pi}_{1}^{1}$ on $\boldsymbol{\Sigma}_{1}^{1}$ and generated by closed sets, then $\mathbb{P}_{\mathcal{I}}$ is proper in all forcing extensions and hence the countable-support iteration of such forcings is proper.

Solecki proved [18, Theorem 1] that if $\mathcal{I}$ is a $\sigma$-ideal on a Polish space $X$ generated by closed sets, then each analytic set in $X$ either belongs to $\mathcal{I}$ or contains an $\mathcal{I}$ positive $\mathbf{G}_{\delta}$ set.

Note that this result has forcing consequences: it implies that if $\mathcal{I}$ is generated by closed sets, then the forcing $\mathbb{P}_{\mathcal{I}}$ is equivalent to the forcing $\mathbb{Q}_{\mathcal{I}}$ with analytic $\mathcal{I}$-positive sets.

Kanovei and Zapletal [22, Theorem 5.1.9] proved that if $\mathcal{I}$ is iterable and $\boldsymbol{\Pi}_{1}^{1}$ on $\boldsymbol{\Sigma}_{1}^{1}$, then for each $\alpha<\omega_{1}$, any analytic set $A \subseteq X^{\alpha}$ either belongs to $\mathcal{I}^{\alpha}$ (the $\alpha$-th Fubini power of $\mathcal{I}$ ) or contains a special kind of Borel $\mathcal{I}^{\alpha}$-positive set. In this paper we extend this result and prove an analogue of the Solecki theorem for the Fubini products of $\boldsymbol{\Pi}_{1}^{1}$ on $\boldsymbol{\Sigma}_{1}^{1} \sigma$-ideals generated by closed sets. Namely, we re-introduce the notion of $\overline{\mathcal{I}}$-positive cubes when $\left\langle\mathcal{I}_{\beta}: \beta<\alpha\right\rangle$ is a sequence of $\sigma$-ideals, $\left\langle X_{\beta}: \beta<\alpha\right\rangle$ is a sequence of Polish spaces, $\mathcal{I}_{\beta}$ on $X_{\beta}$ respectively, and $\alpha$ is a countable ordinal. Similar notions have been considered by other authors under various different names; cf. [8, 4] or [22]. We prove the following (for the definitions see Section (4).

Theorem 1.2. Let $\left\langle X_{n}: n<\omega\right\rangle$ be a sequence of Polish spaces and $\overline{\mathcal{I}}=\left\langle\mathcal{I}_{n}: n<\right.$ $\omega)$ a sequence of $\boldsymbol{\Pi}_{1}^{1}$ on $\boldsymbol{\Sigma}_{1}^{1} \sigma$-ideals generated by closed sets, $\mathcal{I}_{n}$ on $X_{n}$, respectively. If $A \subseteq \prod_{n<\omega} X_{n}$ is $\boldsymbol{\Sigma}_{1}^{1}$, then

- either $A \in \bigotimes_{n<\omega} \mathcal{I}_{n}$

- or $A$ contains an $\overline{\mathcal{I}}$-positive $\mathbf{G}_{\delta}$ cube.

Recall that Sacks and Miller forcings are equivalent to forcings with trees (perfect or superperfect trees, respectively). In both these cases we have fusion (Axiom A), which implies both the properness and continuous reading of names. We generalize this as follows.

Theorem 1.3. If $\mathcal{I}$ is a $\sigma$-ideal generated by closed sets on a Polish space $X$, then the forcing $\mathbb{P}_{\mathcal{I}}$ is equivalent to a forcing with trees with the fusion property.

Axiom A alone can also be deduced from a result of Ishiu [6. Theorem B] and the fact that $\mathbb{P}_{\mathcal{I}}$ is $<\omega_{1}$-proper [23, Lemma 1.3]. Our result shows how to introduce additional structure (trees) which gives deeper insight in the forcing $\mathbb{P}_{\mathcal{I}}$. In particular, it can be used to give an alternative proof of the continuous reading of names.

The proof of Theorem 1.3 uses a technique of fusion games, which are generalizations of the Banach-Mazur game (cf. 9, Section 8.H]). Independently, T. Mátrai 11] introduced and studied similar games. The approach of [11] shows a connection between fusion games and infinite-dimensional perfect set theorems.

In the last two sections we study examples of $\sigma$-ideals generated by closed sets, motivated by analysis and measure theory. 
First, we investigate an example from measure theory. Let $\mathcal{E}$ denote the $\sigma$-ideal generated by closed null sets in the Cantor space (cf. [3]). In Theorem 7.1 we show that $\mathbb{P}_{\mathcal{E}}$ does not add Cohen reals, which implies that any forcing extension with $\mathbb{P}_{\mathcal{E}}$ is minimal. In Corollary 7.4 we establish a fusion game for the $\sigma$-ideal $\mathcal{E}$.

Next we study an example from the theory of real functions. Let $X$ and $Y$ be Polish. To any function $f: X \rightarrow Y$ we associate the $\sigma$-ideal $\mathcal{I}^{f}$ (on $X$ ) generated by closed sets on which $f$ is continuous. The $\sigma$-ideal $\mathcal{I}^{f}$ is nontrivial if and only if $f$ cannot be decomposed into countably many continuous functions with closed domains, i.e. $f$ is not piecewise continuous. Piecewise continuity of Baire class 1 functions has been studied by several authors (e.g. Jayne and Rogers [7, Solecki [19], and Andretta [1]). In Corollary 8.10 we show that if $f: X \rightarrow \omega^{\omega}$ is Baire class 1 and not piecewise continuous, then the forcing $\mathbb{P}_{\mathcal{I}^{f}}$ is equivalent to Miller forcing. As a consequence, in Corollary 8.11 we get that any Borel function defined on an $\mathcal{I}^{f}$-positive set can be restricted to an $\mathcal{I}^{f}$-positive set, on which it is either 1-1 or constant. In Corollary 8.14 we establish a fusion game for the $\sigma$-ideal $\mathcal{I}^{f}$ when $f: 2^{\omega} \rightarrow 2^{\omega}$ is Borel.

\section{Notation}

All Polish spaces in this paper are assumed to be recursively presented.

If $T \subseteq Y^{<\omega}$ is a tree, then we say that $T$ is a tree on $Y$. We write $\lim (T)$ for $\left\{x \in Y^{\omega}:(\forall n<\omega) x\lceil n \in T\}\right.$. $\operatorname{Lev}_{n}(T)$ stands for the set of all elements of $T$ which have length $n$. If $\tau \in T$, then we write $T(\tau)$ for the tree $\{\sigma \in T: \sigma \subseteq \tau \vee \tau \subseteq \sigma\}$. We write $[\tau]_{T}$ for $\{x \in \lim (T): \tau \subseteq x\}$ (when $T$ is clear from the context, such as $\omega^{<\omega}$ or $2^{<\omega}$, then we write only $\left.[\tau]\right)$. We say that $\tau \in T$ is a stem of $T$ if $T=T(\tau)$ and $\tau$ is maximal such. We say that $F \subseteq T$ is a front of $T$ if $F$ is an antichain in $T$ and for each $x \in \lim (T)$ there is $n<\omega$ such that $x\lceil n \in F$.

By a $\sigma$-ideal we mean a family $\mathcal{I} \subseteq \mathcal{P}(X)$ closed under subsets and countable unions. We say that a set $B \subseteq X$ is $\mathcal{I}$-positive if $B \notin \mathcal{I}$. A $\sigma$-ideal of analytic sets is a family of analytic sets closed under analytic subsets and countable unions. A $\sigma$-ideal of closed sets is defined analogously. If $\mathcal{I}$ is a $\sigma$-ideal and $B$ is an $\mathcal{I}$-positive set, then we write $\mathcal{I} \mid B$ for $\{A \cap B: A \in \mathcal{I}\}$.

\section{Coanalytic families of Closed Sets}

If $\mathcal{K}$ is a family of closed subsets of a Polish space $X$, then its projective complexity can be defined in terms of the Effros space $F(X)$. Namely, if $\boldsymbol{\Gamma}$ is a projective pointclass, then we say that $\mathcal{K}$ is $\boldsymbol{\Gamma}$ if it belongs to $\boldsymbol{\Gamma}$ in $F(X)$.

Recall that if $X=\omega^{\omega}$, then for each closed set $C \subseteq X$ there is a pruned subtree $T$ of $\omega^{<\omega}$ such that $C=\lim (T)$. If $X$ is an arbitrary Polish space, then for each closed set $C \subseteq X$ the family $\mathcal{U}=\{U$ basic open : $U \cap C=\emptyset)\}$ can be treated as a code for $C$ (since $C=X \backslash \cup \mathcal{U}$ ). Moreover, the family $\mathcal{U}$ has the following property:

$$
\forall U \text { basic open } U \subseteq \bigcup \mathcal{U} \Rightarrow U \in \mathcal{U} \text {. }
$$

We can code all families $\mathcal{U}$ satisfying $(*)$ by elements of $\omega^{\omega}$ and create a universal closed set $\tilde{C} \subseteq \omega^{\omega} \times X$ such that if $t \in \omega^{\omega} \operatorname{codes} \mathcal{U}(t)$, then $\tilde{C}_{t}=X \backslash \bigcup \mathcal{U}(t)$.

Using the property $(*)$ of the coding, we can check that the function

$$
\omega^{\omega} \ni t \mapsto \tilde{C}_{t} \in F(X)
$$


is Borel measurable (i.e. preimages of Borel sets in $F(X)$ are Borel). Therefore, for any projective pointclass $\boldsymbol{\Gamma}$, a family of closed sets $\mathcal{K}$ is $\boldsymbol{\Gamma}$ if and only if the set $\left\{t \in \omega^{\omega}: \tilde{C}_{t} \in \mathcal{K}\right\}$ is $\boldsymbol{\Gamma}$ in $\omega^{\omega}$.

The projective complexity of families of closed subsets of $X$ can also be generalized to families of sets in other Borel pointclasses - in terms of universal sets. We will now introduce a Borel structure on the family of $\mathbf{G}_{\delta}$ sets.

Note that for any $\mathbf{G}_{\delta}$ set $G \subseteq \omega^{\omega}$ there is a pruned tree $T \subseteq \omega^{<\omega}$ and a family $\left\langle\sigma_{\tau} \in \omega^{<\omega}: \tau \in T\right\rangle$ such that the family of clopen sets $\left\langle\left[\sigma_{\tau}\right]: \tau \in T\right\rangle$ forms a Lusin scheme and $G=\bigcap_{n<\omega} \bigcup_{\tau \in T \cap \omega^{n}}\left[\sigma_{\tau}\right]$. Generalizing this to an arbitrary Polish space $X$ we claim that for any $\mathbf{G}_{\delta}$ set $G$ in $X$ there is a Souslin scheme $\left\langle U_{\tau}: \tau \in \omega^{<\omega}\right\rangle$ of basic open sets such that

(i) $\operatorname{diam}\left(U_{\tau}\right)<1 /|\tau|$,

(ii) $\overline{U_{\tau}} \subseteq U_{\tau \uparrow(|\tau|-1)}$,

(iii) if $U_{\tau} \neq \emptyset$, then $U_{\tau^{\wedge} n} \neq \emptyset$ for some $n<\omega$,

and $G=\bigcap_{n<\omega} \bigcup_{|\tau|=n} U_{\tau}$. Indeed, if $G=\bigcap_{n<\omega} O_{n}$ (each $O_{n}$ open and $O_{n+1} \subseteq$ $O_{n}$ ), then we construct a Souslin scheme $U_{\tau}$ by induction on $|\tau|$ as follows. Having all $U_{\tau}$ for $|\tau| \leq n$ we find a family $\left\{U_{\tau}: \tau \in \omega^{n+1}\right\}$ such that

- for each $\tau \in \omega^{n+1}$ we have $U_{\tau} \cap G \neq \emptyset$,

- for each $\sigma \in \omega^{n}$ we have $U_{\sigma} \cap O_{n+1}=\bigcup\left\{\overline{U_{\tau}}: \sigma \subseteq \tau, \tau \in \omega^{n+1}\right\}$.

Let us code all Souslin schemes of clopen sets satisfying (i)-(iii) by elements of the Baire space $\omega^{\omega}$ and create a universal $\mathbf{G}_{\delta}$ set $\tilde{G} \subseteq \omega^{\omega} \times X$ such that if $t \in \omega^{\omega}$ codes a Souslin scheme $\left\langle U_{\tau}(t): \tau \in \omega^{<\omega}\right\rangle$, then $\tilde{G}_{t}=\bigcap_{n<\omega} \bigcup_{|\tau|=n} U_{\tau}(t)$.

Remark 3.1. Here we show how the above coding is done in the case of the Baire space and Luzin schemes (the general case is analogous). We pick any bijection $\rho$ between $\omega$ and $\omega^{<\omega}$ and consider the set $H$ of all elements of $\omega^{\omega}$ which code (via $\rho$ ) a Luzin scheme satisfying (iii). This set is a $\mathbf{G}_{\delta}$ set and thus there is a continuous bijection $f: \omega^{\omega} \rightarrow H$. Now, we say that $x \in \omega^{\omega}$ codes a $\mathbf{G}_{\delta}$ set $G$ if $f(x)$ codes a Luzin scheme $U_{\tau}$ such that $G=\bigcap_{n<\omega} \bigcup_{|\tau|=n} U_{\tau}$.

Lemma 3.2. If $U \subseteq X$ is open, then

$$
\left\{t \in \omega^{\omega}: \tilde{G}_{t} \cap U \neq \emptyset\right\} \text { is open. }
$$

Proof. Note that by (iii) and (ii), $\tilde{G}_{t} \cap U \neq \emptyset$ if and only if there is a nonempty basic open set $V \subseteq U$ such that $V$ occurs in the Souslin scheme coded by $t$.

If $\boldsymbol{\Gamma}$ is a projective pointclass and $\mathcal{G}$ is a family of $\mathbf{G}_{\delta}$ sets, then we say that $\mathcal{G}$ is $\boldsymbol{\Gamma}$ if $\left\{t \in \omega^{\omega}: \tilde{G}_{t} \in \mathcal{G}\right\}$ is $\boldsymbol{\Gamma}$ in $\omega^{\omega}$. By Lemma 3.2 the map

$$
\mathbf{G}_{\delta} \ni G \mapsto \bar{G} \in F(X)
$$

is Borel (i.e. preimages of Borel sets in $F(X)$ are Borel).

Let $\mathcal{K}$ be a family of closed sets in a Polish space $X$. We say that $\mathcal{K}$ is hereditary if for any two closed sets $C, D$ such that $C \subseteq D$, if $D \in \mathcal{K}$, then $C \in \mathcal{K}$.

Let $\mathcal{I}$ be a $\sigma$-ideal on a Polish space $X$ and $A \subseteq X$. We say that $A$ is $\mathcal{I}$-perfect if $A \neq \emptyset$ and for each open set $U$ the set $A \cap U$ is either empty or $\mathcal{I}$-positive. If $\mathcal{K}$ is a family of closed sets in a Polish space $X$ and $D \subseteq X$ is closed, then we say that $D$ is $\mathcal{K}$-perfect if the sets from $\mathcal{K}$ have relatively empty interior on $D$. Note that if $\mathcal{K}$ is hereditary, then a closed set $D$ is $\mathcal{K}$-perfect if and only if for each basic open set $U$ in $X$, either $U \cap D=\emptyset$ or $\overline{U \cap D} \notin \mathcal{K}$. 
Lemma 3.3. Let $\mathcal{I}$ be a $\sigma$-ideal generated by closed sets on a Polish space $X$. If $G \subseteq \omega^{\omega}$ is $a \mathbf{G}_{\delta}$ set and $\bar{G}$ is $\mathcal{I}$-perfect, then $G \notin \mathcal{I}$.

Proof. Let $C=\bar{G}$ and suppose $C$ is $\mathcal{I}$-perfect yet $G \in \mathcal{I}$. If $G \subseteq \bigcup_{n} F_{n}$ and $F_{n}$ are closed sets in $\mathcal{I}$, then each $F_{n} \cap C$ is a closed nowhere dense subset of $C$. This contradicts the Baire category theorem.

Lemma 3.4. Let $\mathcal{I}$ be a $\sigma$-ideal generated by closed sets on a Polish space $X$. If $G \subseteq \omega^{\omega}$ is an $\mathcal{I}$-positive $\mathbf{G}_{\delta}$ set, then it contains an $\mathcal{I}$-perfect $\mathbf{G}_{\delta}$ set $G^{\prime}$.

Proof. Put $G^{\prime}=G \backslash \bigcup\{U: U$ is basic open set and $U \cap G \in \mathcal{I}\}$.

Lemma 3.5. Let $X$ be a Polish space.

(i) Let $\mathcal{I}$ be a $\sigma$-ideal on $X$ generated by closed sets. If $G \subseteq X$ is a $\mathbf{G}_{\delta}$ set, then $G$ is $\mathcal{I}$-perfect if and only if $\bar{G}$ is $\mathcal{I}$-perfect.

(ii) Let $\mathcal{K}$ be a family of closed subsets of $X$, let $\sigma(\mathcal{K})$ be the $\sigma$-ideal of closed sets generated by $\mathcal{K}$ and let $\mathcal{I}$ be the $\sigma$-ideal generated by $\mathcal{K}$. If $D \subseteq X$ is closed, then the following are equivalent:

- $D$ is $\mathcal{K}$-perfect,

- $D$ is $\sigma(\mathcal{K})$-perfect,

- $D$ is $\mathcal{I}$-perfect.

Proof. (i) Clearly, if $G$ is $\mathcal{I}$-perfect, then $\bar{G}$ is also $\mathcal{I}$-perfect. Suppose $\bar{G}$ is $\mathcal{I}$-perfect but $G$ is not $\mathcal{I}$-perfect. Then we can find an open set $U$ such that $U \cap G \in \mathcal{I}$ and $U \cap G \neq \emptyset$. Consider $U \cap \bar{G}$, which is $\mathcal{I}$-perfect because $\bar{G}$ is $\mathcal{I}$-perfect. $U \cap \bar{G}$ is a Polish $\mathcal{I}$-perfect space which contains a dense $\mathbf{G}_{\delta}$ set in $\mathcal{I}$. By Lemma 3.3 we get a contradiction with the Baire category theorem.

(ii) This follows directly from the Baire category theorem.

Lemma 3.6. Let $X$ be a Polish space and let $\mathcal{I}$ be a $\boldsymbol{\Pi}_{1}^{1}$ on $\boldsymbol{\Sigma}_{1}^{1} \sigma$-ideal generated by closed sets on $X$. Let $\mathcal{K}$ be a hereditary coanalytic family of closed sets on $X$. Then

(i) the family of $\mathcal{I}$-perfect $\mathbf{G}_{\delta}$ sets is $\boldsymbol{\Sigma}_{1}^{1}$,

(ii) the family of $\mathcal{K}$-perfect closed sets is $\boldsymbol{\Sigma}_{1}^{1}$,

(iii) the family of $\mathbf{G}_{\delta}$ sets with $\mathcal{K}$-perfect closure is $\boldsymbol{\Sigma}_{1}^{1}$.

Proof. (i) We see that $G \in \mathbf{G}_{\delta}$ is $\mathcal{I}$-perfect if and only if

$$
G \neq \emptyset \wedge \forall U \text { basic open }(G \cap U \neq \emptyset \Rightarrow G \cap U \notin \mathcal{I}) .
$$

This is a $\boldsymbol{\Sigma}_{1}^{1}$ condition by Lemma 3.2 and the assumption that $\mathcal{I}$ is $\boldsymbol{\Pi}_{1}^{1}$ on $\boldsymbol{\Sigma}_{1}^{1}$.

(ii) Note that a closed set $D$ is $\mathcal{K}$-perfect if and only if

$$
D \neq \emptyset \wedge \forall U \text { basic open }(D \cap U \neq \emptyset \Rightarrow \overline{D \cap U} \notin \mathcal{K}) .
$$

This is a $\boldsymbol{\Sigma}_{1}^{1}$ condition since the closure is a Borel map.

(iii) This follows (ii) and the fact that the closure is a Borel map.

Remark 3.7. In addition to coding $\mathbf{G}_{\delta}$ sets by Luzin schemes, we can also code continuous partial functions on their dense $\mathbf{G}_{\delta}$ subsets, that is, triples $\left(G, f, G^{\prime}\right)$ where $G$ is a $\mathbf{G}_{\delta}$ set, $G^{\prime}$ is a dense $\mathbf{G}_{\delta}$ subset of $G$ and $f: G^{\prime} \rightarrow \omega^{\omega}$ is continuous. For a sample method of coding see e.g. [9, Proposition 2.6]. If $D \subseteq \omega^{\omega} \times \omega^{\omega}$ is a closed set and $G \subseteq \omega^{\omega}$ is a $\mathbf{G}_{\delta}$ set, then we write

$$
f: G \stackrel{*}{\rightarrow} D
$$


to denote that $f$ is a continuous function from a dense $\mathbf{G}_{\delta}$ subset of $G$ and the graph of $f$ is contained in $D$.

It is well known (see [9, Theorem 35.38] or [5, Lemma 4.8]) that if $\mathcal{K}$ is a coanalytic hereditary family of closed sets, then the $\sigma$-ideal generated by $\mathcal{K}$ is $\boldsymbol{\Pi}_{1}^{1}$ on $\boldsymbol{\Sigma}_{1}^{1}$. Let us present a new proof of this fact, which uses idealized forcing and Solecki's theorem.

Corollary 3.8. Let $X$ be a Polish space. If $\mathcal{K}$ is a coanalytic hereditary family of closed sets in $X$, then the $\sigma$-ideal generated by $\mathcal{K}$ is $\boldsymbol{\Pi}_{1}^{1}$ on $\boldsymbol{\Sigma}_{1}^{1}$.

Proof. Let $\mathcal{I}$ be the $\sigma$-ideal generated by $\mathcal{K}$ and let $A \subseteq X \times X$ be $\Sigma_{1}^{1}$. Denote by $\mathcal{G}$ the family of $\mathcal{I}$-perfect $\mathbf{G}_{\delta}$ sets. By Lemmas 3.5 and 3.6, $\mathcal{G}$ is $\boldsymbol{\Sigma}_{1}^{1}$. By Solecki's theorem and Lemma 3.4 if $x \in X$, then

$$
A_{x} \notin \mathcal{I} \quad \text { iff } \quad \exists G \in \mathcal{G} \quad G \subseteq A_{x} .
$$

Let $D \subseteq X^{2} \times \omega^{\omega}$ be a closed set such that $A=\pi[D](\pi$ denotes the projection to the first two coordinates). Note that $G \subseteq A_{x}$ is equivalent to

$$
\forall y \in G \exists z \in \omega^{\omega}(y, z) \in D_{x} .
$$

By $\boldsymbol{\Sigma}_{2}^{1}$-absoluteness we get a name $\dot{z}_{x}$ such that

$$
G \Vdash\left(\dot{g}, \dot{z}_{x}\right) \in D_{x} .
$$

Now, by the continuous reading of names and properness of $\mathbb{P}_{\mathcal{I}}$ we get a $G^{\prime} \in \mathcal{G}$, $G^{\prime} \subseteq G$ and a continuous function $f_{x}: G^{\prime} \rightarrow \omega^{\omega}$ reading $\dot{z}_{x}$. Notice that the graph of $f_{x}$ is contained in $D_{x}$, so $f_{x}: G^{\prime} \stackrel{*}{\rightarrow} D_{x}$. Conversely, if there is such a function $f$, then $\operatorname{dom}(f)$ is an $\mathcal{I}$-perfect $\mathbf{G}_{\delta}$-set contained in $A_{x}$. Thus, we have shown that

$$
A_{x} \notin \mathcal{I} \quad \text { iff } \quad \exists G \in \mathcal{G} \quad \exists f: G \stackrel{*}{\rightarrow} D_{x} .
$$

Using the coding of $\mathbf{G}_{\delta}$ sets and partial continuous functions, one can easily check that

$$
\exists f: G \stackrel{*}{\rightarrow} D_{x}
$$

is a $\boldsymbol{\Sigma}_{1}^{1}$ formula. Thus, the whole formula is $\boldsymbol{\Sigma}_{1}^{1}$ and we are done.

Analytic sets in a Polish space $X$ can be coded by a $\Sigma_{1}^{1}$-universal $\Sigma_{1}^{1}$ set on $\omega^{\omega} \times X$. In the remaining part of this section we fix a universal analytic set $\tilde{A} \subseteq \omega^{\omega} \times X$ which is $\Sigma_{1}^{1}$ and good (cf. [12, Section 3.H.1]). The set $\tilde{A}$ will be used to code analytic sets in $X$ as well as $\Sigma_{1}^{1}(t)$ sets for each $t \in \omega^{\omega}$.

We say that a set $S \subseteq \omega^{\omega}$ codes a $\sigma$-ideal $\mathcal{I}$ of analytic sets if the family $\mathcal{I}=$ $\left\{\tilde{A}_{t}: t \in S\right\}$ is a $\sigma$-ideal of analytic sets. Let $I(v)$ be a $\Pi_{1}^{1}$ formula. Note that the family of analytic sets whose codes satisfy $I(v)$ is $\boldsymbol{\Pi}_{1}^{1}$ on $\boldsymbol{\Sigma}_{1}^{1}$ (because $\tilde{A}$ is good and $I(v)$ is a $\Pi_{1}^{1}$ formula).

Lemma 3.9 (Folklore). If $\mathcal{A}$ is a $\Pi_{1}^{1}$ on $\boldsymbol{\Sigma}_{1}^{1}$ family of analytic sets, then $\mathcal{A}$ is downward closed, i.e. if $A, B \in \boldsymbol{\Sigma}_{1}^{1}$ are such that $A \subseteq B$ and $B \in \mathcal{A}$, then $A \in \mathcal{A}$.

Proof. Suppose $A \subseteq B$ are $\boldsymbol{\Sigma}_{1}^{1}$ and $B \in \mathcal{A}$. Let $Z \subseteq \omega^{\omega}$ be such that $Z \in \Sigma_{1}^{1} \backslash \boldsymbol{\Pi}_{1}^{1}$. Take $L \subseteq \omega^{\omega} \times X$ such that

$$
(t, x) \in L \quad \Leftrightarrow \quad(t \in Z \wedge x \in B) \vee x \in A .
$$

As $\left\{t \in \omega^{\omega}: L_{t} \in \mathcal{A}\right\} \in \Pi_{1}^{1}$, we conclude that $A \in \mathcal{A}$. 
Suppose $V \subseteq W$ is a generic extension and in $V$ we have a $\boldsymbol{\Pi}_{1}^{1}$ on $\boldsymbol{\Sigma}_{1}^{1} \sigma$-ideal $\mathcal{I}$. Let $I(v)$ be a $\bar{\Pi}_{1}^{1}$ formula which codes the $\sigma$-ideal of analytic sets $\mathcal{I} \cap \boldsymbol{\Sigma}_{1}^{1}$. By $\mathcal{I}^{W}$ we denote the family of analytic sets whose codes satisfy $I(v)$ in $V[G]$. This definition does not depend on the formula $I(v)$ since if $I^{\prime}(v)$ is another such formula, then

$$
\forall t \in \omega^{\omega} \quad I(t) \Leftrightarrow I^{\prime}(t)
$$

is a $\Pi_{2}^{1}$ sentence and hence it is absolute for $V \subseteq W$.

Now we prove Theorem 1.1 .

Proof of Theorem 1.1, Let $K(v)$ be a $\boldsymbol{\Pi}_{1}^{1}$ formula defining the set of codes of closed sets in $\mathcal{I}$. By $\mathcal{K}^{W}$ we denote the family of closed sets in $W$ whose codes satisfy $K(v)$ (as previously, this does not depend on the formula $K(v)$ ).

First we show that in $W$ the family $\mathcal{K}^{W}$ is hereditary. Consider the following sentence:

$$
\exists t, s \in \omega^{\omega} \quad\left(\neg K(s) \wedge K(t) \wedge \tilde{C}_{s} \subseteq \tilde{C}_{t}\right) .
$$

It is routine to check that it is $\boldsymbol{\Sigma}_{2}^{1}$ and hence absolute for $V \subseteq W$. This shows that $\mathcal{K}^{W}$ is hereditary.

Next we show that $\mathcal{I}^{W}$ is a $\sigma$-ideal of analytic sets. Let $D \subseteq \omega^{\omega} \times X \times \omega^{\omega}$ be a closed set such that $\pi[D]=\tilde{A}$ (here $\pi$ denotes the projection to the first two coordinates). Consider the following formula $I^{\prime}(v)$ :

$$
\neg\left(\exists G \in \mathbf{G}_{\delta} \quad \bar{G} \text { is } K \text {-perfect } \wedge \exists f: G \stackrel{*}{\rightarrow} D_{v}\right)
$$

(writing that $\bar{G}$ is $K$-perfect we mean that $\bar{G}$ is perfect with respect to the family of closed sets defined by $K(v)$ ). Using Lemma 3.6(iii) we can check that $I^{\prime}(v)$ is a $\Pi_{1}^{1}$ formula. From the proof of Corollary 3.8 and from Lemmas 3.3, 3.4 and 3.5 we conclude that in $V$ we have

$$
\forall t \in \omega^{\omega} \quad I(t) \Leftrightarrow I^{\prime}(t) .
$$

This is a $\Pi_{2}^{1}$ sentence and hence it holds in $W$. Therefore, it is enough to check that $\left(I^{\prime}\right)^{W}\left(\omega^{\omega}\right)$ codes a $\sigma$-ideal of analytic sets. However, it follows from Solecki's theorem and from Lemmas 3.3, 3.4 and 3.5, that $\left(I^{\prime}\right)^{W}\left(\omega^{\omega}\right)$ codes the $\sigma$-ideal generated by $K\left(\omega^{\omega}\right)^{W}$.

The fact that $\mathcal{I}^{W}$ is $\boldsymbol{\Pi}_{1}^{1}$ on $\boldsymbol{\Sigma}_{1}^{1}$ now follows from the remarks preceding this proposition.

We also have the following alternative proof.

Alternative proof of Theorem 1.1. Throughout this proof we denote the closure of a set $A$ by $\operatorname{cl} A$. Without loss of generality assume that $\mathcal{I}$ is $\Pi_{1}^{1}$ on $\Sigma_{1}^{1}$ and $X=\omega^{\omega}$.

We will use the following notation. If $\varphi(v)$ is a formula and $t \in \omega^{\omega}$, then by $\Sigma_{1}^{1}(t) \wedge \varphi\left(\right.$ respectively $\left.\Delta_{1}^{1}(t) \wedge \varphi\right)$ we denote the family of $\Sigma_{1}^{1}(t)$ (respectively $\Delta_{1}^{1}(t)$ ) sets whose codes satisfy $\varphi(v)$.

Let $I(v)$ be a $\Pi_{1}^{1}$ formula defining the set of codes of analytic sets in $\mathcal{I}$. Let $\mathcal{K}$ be the family of closed sets in $\mathcal{I}$ and let $K(v)$ be a $\Pi_{1}^{1}$ formula defining the set of codes of the (closed) sets in $\mathcal{K}$ (in terms of the universal closed set $\tilde{C}$ ). Consider the formula $\hat{K}(v)$ saying that $\operatorname{cl} \tilde{A}_{v} \in \mathcal{K}$. Note that $\hat{K}(v)$ can be written as follows:

$$
\forall s \in \omega^{\omega} \quad \tilde{C}_{s} \subseteq \operatorname{cl} \tilde{A}_{v} \Rightarrow K(s),
$$

and notice that it is a $\Pi_{1}^{1}$ formula. 
Consider the set $F \subseteq \omega^{\omega} \times X$ defined as follows:

$$
(t, x) \in F \quad \text { iff } \quad x \in \bigcup\left(\Delta_{1}^{1}(t) \wedge \hat{K}\right) .
$$

By the usual coding of $\Delta_{1}^{1}$ sets we get that $F$ is $\Pi_{1}^{1}$.

Lemma 3.10. For each $t \in \omega^{\omega}$ we have

$$
\bigcup\left(\Sigma_{1}^{1}(t) \wedge \hat{K}\right)=\bigcup\left(\Delta_{1}^{1}(t) \wedge \hat{K}\right)=\bigcup\left(\Sigma_{1}^{1}(t) \wedge I\right) .
$$

Proof. Without loss of generality assume that $t=0$. The first equality follows from the First Reflection Theorem (since $\hat{K}(v)$ is a $\Pi_{1}^{1}$ formula). Denote $C=$ $\bigcup\left(\Delta_{1}^{1}(t) \wedge \hat{K}\right)=\bigcup\left(\Sigma_{1}^{1}(t) \wedge \hat{K}\right)$.

In the second equality, the left-to-right inclusion is obvious since $\hat{K}(s)$ implies $I(s)$, for each $s \in \omega^{\omega}$. We need to prove that if $A \in \Sigma_{1}^{1}$ is not contained in $C$, then $A \notin \mathcal{I}$. Suppose $A \in \Sigma_{1}^{1}$ and $A \nsubseteq \subset C$. Since $C \in \Pi_{1}^{1}$, we may assume that $A \cap C=\emptyset$. Let $T$ be a recursive pruned tree on $\omega \times \omega$ such that $A=\operatorname{proj}[T]$. If $A \in \mathcal{I}$, then there is a sequence of closed sets $\left\langle D_{n}: n<\omega\right\rangle$ such that each $D_{n} \in \mathcal{I}$ and $A \subseteq \bigcup_{n<\omega} D_{n}$. By induction we construct a sequence of $\left\langle\tau_{n} \in \omega^{<\omega}\right\rangle$ and $\sigma_{n} \in T$ such that for each $n<\omega$ the following hold:

- $\sigma_{n+1} \supsetneq \sigma_{n}$ and $\tau_{n+1} \supsetneq \tau_{n}$,

- $\operatorname{proj}\left[T\left(\sigma_{n}\right)\right] \subseteq\left[\tau_{n}\right]$,

- $\operatorname{proj}\left[T\left(\sigma_{n-1}\right)\right] \cap\left[\tau_{n}\right] \cap D_{n}=\emptyset$.

We take $\sigma_{-1}=\emptyset$. Suppose $\sigma_{n}$ and $\tau_{n}$ are constructed. Notice that $\operatorname{proj}\left[T\left(\sigma_{n}\right)\right]$ is $\Sigma_{1}^{1}$. Since $A \cap C=\emptyset$ we see that $\operatorname{cl}\left(\operatorname{proj}\left[T\left(\sigma_{n}\right)\right]\right) \notin \mathcal{K}$. Consequently, $\operatorname{proj}\left[T\left(\sigma_{n}\right)\right] \not \subset$ $D_{n}$ and hence there is $\tau_{n+1} \supsetneq \tau_{n},\left[\tau_{n+1}\right] \subseteq \tau_{n}$ such that

(i) $\operatorname{proj}\left[T\left(\sigma_{n}\right)\right] \cap\left[\tau_{n+1}\right] \neq \emptyset$,

(ii) $\operatorname{proj}\left[T\left(\sigma_{n}\right)\right] \cap\left[\tau_{n+1}\right] \cap D_{n}=\emptyset$.

Using (i) find $\sigma_{n+1} \supsetneq \sigma_{n}$ such that $\sigma_{n+1} \in T$ and $\operatorname{proj}\left[T\left(\sigma_{n+1}\right)\right] \subseteq\left[\tau_{n+1}\right]$.

Now, if $s=\bigcup_{n<\omega} \sigma_{n}$, then $s \in \lim (T)$, so $\pi(s) \in A$, but $\pi(s) \notin \bigcup_{n<\omega} D_{n}$. This ends the proof of the lemma.

Consider the following formula $I^{\prime}(v)$ ( $v$ is a variable):

$$
\forall z \in X \quad z \in \tilde{A}_{v} \Rightarrow z \in F_{v} .
$$

Note that $I^{\prime}$ is a $\Pi_{1}^{1}$ formula and

$$
V \models \forall t \in \omega^{\omega} \quad I(t) \Leftrightarrow I^{\prime}(t) .
$$

This is a $\Pi_{2}^{1}$ sentence, so by absoluteness we see that $I$ and $I^{\prime}$ define the same set of codes of analytic sets in $W$.

Now we will show that $\mathcal{I}^{W}$ is a $\sigma$-ideal generated by closed sets. The fact that $\mathcal{I}^{W}$ is closed under taking analytic subsets follows from Lemma 3.9 because $\mathcal{I}^{W}$ is $\Pi_{1}^{1}$ on $\boldsymbol{\Sigma}_{1}^{1}$.

Let us show that $\mathcal{I}^{W}$ is closed under countable unions. Pick a recursive bijection $\lceil\cdot\rceil:\left(\omega^{\omega}\right)^{\omega} \rightarrow \omega^{\omega}$. The sentence

$$
\forall\left\langle t_{n}: n<\omega\right\rangle \in\left(\omega^{\omega}\right)^{\omega} \quad\left(\left(\forall n<\omega I^{\prime}\left(t_{n}\right)\right) \Rightarrow I^{\prime}\left(\left\lceil t_{n}: n<\omega\right\rceil\right)\right)
$$

is $\Pi_{2}^{1}$ and hence it is absolute. Note that for any $\left\langle t_{n}: n<\omega\right\rangle \in\left(\omega^{\omega}\right)^{\omega}$ we have $F_{t_{k}} \subseteq F_{\left\lceil t_{n}: n<\omega\right\rceil}$ for each $k<\omega$ (because $t_{k} \in \Delta_{1}^{1}\left(\left\lceil t_{n}: n<\omega\right\rceil\right)$ ). Therefore $(*)$ holds in $V$ and hence also in $W$. This shows that the family of analytic sets coded by $\left(I^{\prime}\right)^{W}\left(\omega^{\omega}\right)$ is closed under countable unions. 
To see that $\mathcal{I}^{W}$ is generated by closed sets, take any $t \in W \cap \omega^{\omega}$ such that $\left(\tilde{A}^{W}\right)_{t} \in \mathcal{I}^{W}$. This means that $W \models I^{\prime}(t)$, so $\left(\tilde{A}^{W}\right)_{t} \subseteq\left(F^{W}\right)_{t}$. Let $\left\langle t_{n}: n<\omega\right\rangle \in$ $W$ be the sequence of all elements of $\omega^{\omega}$ in $W$ which are $\Delta_{1}^{1}(t)$ and satisfy $\hat{K}(v)$. By the definition of $F$ we see that

$$
W \models\left(\tilde{A}^{W}\right)_{t} \subseteq \bigcup_{n<\omega}\left(\tilde{A}^{W}\right)_{t_{n}}
$$

is satisfied in $W$. Let $\left\langle s_{n}: n\langle\omega\rangle \in W\right.$ be a sequence of elements of $W \cap \omega^{\omega}$ such that $W \models\left(\tilde{C}^{W}\right)_{s_{n}}=\operatorname{cl}\left(\tilde{A}^{W}\right)_{t_{n}}$ for each $n<\omega$. Now $W \models \hat{K}\left(t_{n}\right)$ implies $W \models K\left(s_{n}\right)$. Therefore $W \models\left(\tilde{C}^{W}\right)_{s_{n}} \in \mathcal{I}^{W}$ because

$$
\forall t, s \in \omega^{\omega} \quad\left(\tilde{A}_{s} \subseteq \tilde{C}_{t} \wedge K(t)\right) \Rightarrow I(s)
$$

is $\Pi_{2}^{1}$ and holds in $V$. Since

$$
W \models\left(\tilde{A}^{W}\right)_{t} \subseteq \bigcup_{n<\omega}\left(\tilde{C}^{W}\right)_{s_{n}},
$$

we conclude that $\mathcal{I}^{W}$ is generated by closed sets.

\section{Products and iterations}

If $\mathcal{I}$ is a $\sigma$-ideal on $X$, then we write $\forall^{\mathcal{I}} x \in X \varphi(x)$ to denote that $\{x \in X$ : $\neg \varphi(x)\} \in \mathcal{I}$. Let $\mathcal{I}$ and $\mathcal{J}$ be $\sigma$-ideals on Polish spaces $X$ and $Y$, respectively. Recall that the Fubini product of $\mathcal{I}$ and $\mathcal{J}$, denoted by $\mathcal{I} \otimes \mathcal{J}$, is the $\sigma$-ideal of those $A \subseteq X \times Y$ such that

$$
\forall^{\mathcal{I}} x \in X \forall^{\mathcal{J}} y \in Y(x, y) \notin A .
$$

If $\mathcal{I}_{k}$ is a $\sigma$-ideal on $X_{k}$, for each $k<n$, then we naturally extend the above definition to define $\bigotimes_{k<n} \mathcal{I}_{k}=\mathcal{I}_{0} \otimes \bigotimes_{0<k<n} \mathcal{I}_{k}$. For each $n<\omega$ we also define the Fubini powers of a $\sigma$-ideal $\mathcal{I}$ as follows: $\mathcal{I}^{n}=\bigotimes_{k<n} \mathcal{I}$.

Lemma 4.1 (Folklore). Suppose $\mathcal{I}$ and $\mathcal{J}$ are $\boldsymbol{\Pi}_{1}^{1}$ on $\boldsymbol{\Sigma}_{1}^{1} \sigma$-ideals on Polish spaces $X$ and $Y$, respectively. Let $A \subseteq X \times Y$ be a $\boldsymbol{\Sigma}_{1}^{1}$ set in $\mathcal{I} \otimes \mathcal{J}$. There is a $\boldsymbol{\Sigma}_{1}^{1}$ set $D$ such that $A \cap D=\emptyset$ and

$$
\forall^{\mathcal{I}} x \in X \forall^{\mathcal{J}} y \in Y \quad(x, y) \in D .
$$

Proof. To simplify notation suppose that $X=Y=\omega^{\omega}, A \in \Sigma_{1}^{1}$, and $\mathcal{I}$ and $\mathcal{J}$ are $\Pi_{1}^{1}$ on $\Sigma_{1}^{1}$. Put

$$
U^{1}=\bigcup\left(\Sigma_{1}^{1} \cap \mathcal{I}\right)
$$

and let $U^{2} \subseteq \omega^{\omega} \times \omega^{\omega}$ be such that for each $t \in \omega^{\omega}$ we have

$$
\left(U^{2}\right)_{t}=\bigcup\left(\Sigma_{1}^{1}(t) \cap \mathcal{J}\right) .
$$

By the First Reflection Theorem we have $U^{1}=\bigcup\left(\Delta_{1}^{1} \cap \mathcal{I}\right)$ and $\left(U^{2}\right)_{t}=\bigcup\left(\Delta_{1}^{1}(t) \cap \mathcal{J}\right)$ for each $t \in \omega^{\omega}$. Therefore, by the usual coding of $\Delta_{1}^{1}$ sets, we get that $U^{1}$ and $U^{2}$ are $\Pi_{1}^{1}$. Put

$$
C=\left(U^{1} \times Y\right) \cup U^{2} .
$$

Notice that $A \subseteq C$ (since otherwise we get that $A \notin \mathcal{I} \otimes \mathcal{J}$ ). Now $B=X \times Y \backslash C$ is as needed. 
Generalizing the finite Fubini products, one can define the Fubini product of length $\alpha$ for any $\alpha<\omega_{1}$. A game-theoretic definition of $\mathcal{I}^{\alpha}$ is given in [22, Definition 5.1.1]. Definition 4.3 below (equivalent to [22, Definition 5.1.1]) appears in [4, p. 74]. If $0<\beta<\alpha$ are countable ordinals, then we write $\pi_{\alpha, \beta}$ for the projection to the first $\beta$ coordinates from $\prod_{\gamma<\alpha} X_{\gamma}$ to $\prod_{\gamma<\beta} X_{\gamma}$. For each $D \subseteq \prod_{\gamma<\alpha} X_{\gamma}$, we define $\pi_{\alpha, 0}[D]$ to be $X$. If $A \subseteq \prod_{\gamma<\beta+1} X_{\gamma}$ and $x \in \prod_{\gamma<\beta} X_{\gamma}$, then $A_{x}$ denotes the vertical section of $A$ at $x$. If $A \subseteq X$ and $x \in X$, then we put $A_{x}=A$.

Definition 4.2. Let $\alpha$ be a countable ordinal, $\left\langle X_{\beta}: \beta<\alpha\right\rangle$ be a sequence of Polish spaces and $\overline{\mathcal{I}}=\left\langle\mathcal{I}_{\beta}: \beta<\alpha\right\rangle$ be a sequence of $\sigma$-ideals, $\mathcal{I}_{\beta}$ on $X_{\beta}$, respectively. We say that a set $D \subseteq \prod_{\beta<\alpha} X_{\beta}$ is an $\overline{\mathcal{I}}$-positive cube if

(i) for each $\beta<\alpha$ and for each $x \in \pi_{\alpha, \beta}[D]$ the set

$$
\left(\pi_{\alpha, \beta+1}[D]\right)_{x} \text { is } \mathcal{I}_{\beta+1} \text {-positive, }
$$

(ii) for each limit $\beta<\alpha$ and $x \in X^{\beta}$,

$$
x \in \pi_{\alpha, \beta}[D] \quad \Leftrightarrow \quad \forall \gamma<\beta \quad x\left\lceil\gamma \in \pi_{\alpha, \gamma}[D] .\right.
$$

We say that $D$ is an $\overline{\mathcal{I}}$-full cube if additionally we have

(i') for each $\beta<\alpha$ and for each $x \in \pi_{\alpha, \beta}[D]$ the set

$$
\left(\pi_{\alpha, \beta+1}[D]\right)_{x} \text { is } \mathcal{I}_{\beta+1} \text {-full. }
$$

If $\boldsymbol{\Gamma}$ is a projective pointclass, then we say that $D$ is an $\overline{\mathcal{I}}$-positive (resp. full) $\boldsymbol{\Gamma}$ cube if $D$ is an $\overline{\mathcal{I}}$-positive (resp. full) cube and additionally

- for each $\beta \leq \alpha$ the set $\pi_{\alpha, \beta}[D] \in \boldsymbol{\Gamma}\left(\prod_{\gamma<\beta} X_{\gamma}\right)$.

Analogous definitions also appear in [8, 4] and [22. Now we define Fubini products.

Definition 4.3. Let $\alpha$ be a countable ordinal, $\left\langle X_{\beta}: \beta<\alpha\right\rangle$ be a sequence of Polish spaces and $\overline{\mathcal{I}}=\left\langle\mathcal{I}_{\beta}: \beta<\alpha\right\rangle$ be a sequence of $\sigma$-ideals, $\mathcal{I}_{\beta}$ on $X_{\beta}$, respectively. A set $B \subseteq \prod_{\beta<\alpha} X_{\beta}$ belongs to $\bigotimes_{\beta<\alpha} \mathcal{I}_{\beta}$ if and only if there is an $\overline{\mathcal{I}}$-full cube $D \subseteq \prod_{\beta<\alpha} X_{\beta}$ disjoint from $B$.

Remark 4.4. Let $X$ be a Polish space and let $\mathcal{I}$ be a $\boldsymbol{\Pi}_{1}^{1}$ on $\boldsymbol{\Sigma}_{1}^{1} \sigma$-ideal on $X$, generated by closed sets. Suppose $A \subseteq X^{2}$ is $\boldsymbol{\Sigma}_{1}^{1}$. We will show that either $A$ belongs to $\mathcal{I}^{2}$ or contains an $\mathcal{I}^{2}$-positive $\mathbf{G}_{\delta}$ set.

Let $D \subseteq X^{2} \times \omega^{\omega}$ be a closed set such that $\pi[D]=A$ (here $\pi$ denotes the projection to the first two coordinates). By Lemma 3.6, the family $\mathcal{G}$ of $\mathcal{I}$-perfect $\mathbf{G}_{\delta}$ sets is $\boldsymbol{\Sigma}_{1}^{1}$ (in the sense of Section 3 in terms of $\tilde{G}$ ). Put $A^{\prime}=\left\{x \in X: A_{x} \notin \mathcal{I}\right\}$. If $A^{\prime} \in \mathcal{I}$, then clearly $A \in \mathcal{I}^{2}$. Suppose that $A^{\prime} \notin \mathcal{I}$.

By Solecki's theorem, for each $x \in A^{\prime}$ there is an $\mathcal{I}$-perfect $\mathbf{G}_{\delta}$ set $G$ contained in $A_{x}$. Pick $x \in A^{\prime}$ and such a $G \subseteq A_{x}$. Using $\boldsymbol{\Sigma}_{2}^{1}$-absoluteness, we get a $\mathbb{P}_{\mathcal{I}}$-name $\dot{y}$ for an element of $D$ such that (we identify $\left(X^{2}\right)_{x}$ with $X$ here)

$$
G \Vdash \pi(\dot{y})=\dot{g}
$$

( $\dot{g}$ is the name for the generic point).

Now, by properness and continuous reading of names for $\mathbb{P}_{\mathcal{I}}$, there is an $\mathcal{I}$-perfect $\mathbf{G}_{\delta}$ set $G^{\prime} \subseteq G$ and a continuous function $f: G^{\prime} \rightarrow \omega^{\omega}$ with $f \subseteq D$. To see this, find a continuous function $f^{\prime}$ reading $\dot{y}$, take a suitable countable elementary submodel $M \prec H_{\kappa}$ ( $\kappa$ large enough), find $G^{\prime}$ consisting of generic reals over $M$ and put 
$f=f^{\prime} \uparrow G$. The fact that $(x, f(x)) \in D$ for $x \in G$ follows from $\Sigma_{1}^{1}$-absoluteness between $M[x]$ and $V$.

Therefore, for each $x \in A^{\prime}$ the following holds:

$$
\exists G \in \mathcal{G} \quad \exists f: G \stackrel{*}{\rightarrow} D_{x} .
$$

This is a $\boldsymbol{\Sigma}_{1}^{1}$ formula, so by $\boldsymbol{\Sigma}_{2}^{1}$-absoluteness we have

$$
A^{\prime} \Vdash \exists G \in \mathcal{G} \quad \exists f: G \stackrel{*}{\rightarrow} D_{\dot{g}} .
$$

Again, by properness and continuous reading of names (applied to the name for (a code of) $\operatorname{dom}(f))$ we get an $\mathcal{I}$-perfect $\mathbf{G}_{\delta}$ set $G^{\prime} \subseteq A^{\prime}$ and a continuous function $g: G^{\prime} \rightarrow \mathcal{G}$ such that for each $x \in G^{\prime}$ we have $\tilde{G}_{g(x)} \subseteq A_{x}$. Let $G=\left\{(x, y) \in X^{2}\right.$ : $\left.x \in G^{\prime} \wedge y \in \tilde{G}_{g(x)}\right\}=(g, i d)^{-1}[\tilde{G}]$. This is an $\mathcal{I}^{2}$-positive $\mathbf{G}_{\delta}$ set contained in $A$.

Note that the following lemma immediately follows from Lemma 4.1 ,

Lemma 4.5 (Folklore). Suppose $\overline{\mathcal{I}}=\left\langle\mathcal{I}_{k}: k\langle n\rangle\right.$ is a sequence of $\boldsymbol{\Pi}_{1}^{1}$ on $\boldsymbol{\Sigma}_{1}^{1}$ $\sigma$-ideals, $\mathcal{I}_{k}$ on $X_{k}$. Let $A \subseteq \prod_{k<n} X_{k}$ be a $\Sigma_{1}^{1}$ set in $\bigotimes_{k<n} \mathcal{I}_{k}$. There is an $\overline{\mathcal{I}}$-full $\Sigma_{1}^{1}$ cube $D$ disjoint from $A$.

If $\alpha$ is a countable ordinal and $\left\langle\mathcal{I}_{\beta}: \beta<\alpha\right\rangle$ is a sequence of iterable $\sigma$-ideals, $\mathcal{I}_{\beta}$ on $X_{\beta}$, then we denote by $*_{\beta<\alpha} \mathbb{P}_{\mathcal{I}_{\beta}}$ the countable support iteration of $\mathbb{P}_{\mathcal{I}_{\beta}}$ 's of length $\alpha$. If $A \subseteq \prod_{\beta<\alpha} X_{\beta}$ is an $\overline{\mathcal{I}}$-positive Bor cube, then we associate with $A$ the following condition $p_{\alpha}(A)$ in $*_{\beta<\alpha} \mathbb{P}_{\mathcal{I}_{\beta}}$. If $\beta<\alpha$, then $p_{\alpha}(A)(\beta)$ is a $*_{\gamma<\beta} \mathbb{P}_{\mathcal{I}_{\gamma}}$-name $\dot{Y}_{\beta}$ (for an $\mathcal{I}_{\beta}$-positive Borel set) such that

$$
p_{\beta}\left(\pi_{\alpha, \beta}[A]\right) \Vdash \dot{Y}_{\beta}=A_{\dot{g}_{\beta}},
$$

where $\dot{g}_{\beta}$ is the name for the $*_{\gamma<\beta} \mathbb{P}_{\mathcal{I}_{\gamma}}$-generic point in $\prod_{\gamma<\beta} X_{\gamma}$. Zapletal proved the following (the statement in [22, Theorem 5.1.6] deals with just one $\sigma$-ideal $\mathcal{I}$, but the proof shows the stronger statement).

Theorem 4.6 (Zapletal, 22, Theorem 5.1.6]). Let $\alpha$ be a countable ordinal. If $\overline{\mathcal{I}}=\left\langle\mathcal{I}_{\beta}: \beta<\alpha\right\rangle$ is a sequence of iterable $\sigma$-ideals on Polish spaces $X_{\beta}$, respectively, then the function $p_{\alpha}$ is a dense embedding from the poset of $\overline{\mathcal{I}}$-positive $\mathbf{B o r}$ cubes (ordered by inclusion) into $*_{\beta<\alpha} \mathbb{P}_{\mathcal{I}_{\beta}}$. Moreover, any $\bigotimes_{\beta<\alpha} \mathcal{I}_{\beta}$-positive Borel set in $\prod_{\beta<\alpha} X_{\beta}$ contains an $\overline{\mathcal{I}}$-positive Bor cube and the forcing $\mathbb{P}_{\bigotimes_{\beta<\alpha} \mathcal{I}_{\beta}}$ is equivalent to $*_{\beta<\alpha} \mathbb{P}_{\mathcal{I}_{\beta}}$.

The proof of this result uses Shelah's method of showing that the countablesupport iteration of proper forcings is proper. Theorem 4.6 was further used by Kanovei and Zapletal to prove the following result (again, the statement of [22, Theorem 5.1.9] deals with one $\sigma$-ideal, but the proof generalizes to the statement below).

Theorem 4.7 (Kanovei, Zapletal, 22, Theorem 5.1.9]). Let $\alpha$ be a countable ordinal and $\overline{\mathcal{I}}=\left\langle\mathcal{I}_{\beta}: \beta\langle\alpha\rangle\right.$ be a sequence of iterable $\sigma$-ideals on Polish spaces $X_{\beta}$, respectively. If $A \subseteq \prod_{\beta<\alpha} X_{\beta}$ is $\Sigma_{1}^{1}$, then either $A \in \bigotimes_{\beta<\alpha} \mathcal{I}_{\beta}$ or $A$ contains an $\overline{\mathcal{I}}$-positive Bor cube.

In the proof of Theorem 4.7. Kanovei and Zapletal generalized Lemma 4.5 in the following way. 
Theorem 4.8 (Kanovei, Zapletal, [22, proof of Theorem 5.1.9]). Let $\alpha$ be a countable ordinal and $\overline{\mathcal{I}}=\left\langle\mathcal{I}_{\beta}: \beta<\alpha\right\rangle$ be a sequence of $\boldsymbol{\Pi}_{1}^{1}$ on $\boldsymbol{\Sigma}_{1}^{1} \sigma$-ideals on Polish spaces $X_{\beta}$, respectively. If $A \subseteq \prod_{\beta<\alpha} X_{\beta}$, is $\boldsymbol{\Sigma}_{1}^{1}$ and $A \in \bigotimes_{\beta<\alpha} \mathcal{I}_{\beta}$, then there is an $\overline{\mathcal{I}}$-full $\boldsymbol{\Sigma}_{1}^{1}$ cube $D$ disjoint from $A$.

The following (unpublished) corollary was communicated to the author by Pawlikowski.

Corollary 4.9 (Pawlikowski, 15]). If $X$ is a Polish space and $\alpha$ is a countable ordinal, then $\mathcal{M}(X)^{\alpha} \cap \Sigma_{1}^{1}\left(X^{\alpha}\right) \subseteq \mathcal{M}\left(X^{\alpha}\right)$.

Theorem 1.2 was motivated by Solecki's theorem, Theorem 4.7 and Corollary 4.9. Now we restate it, in a slightly stronger version.

Theorem 4.10. Let $\left\langle X_{n}: n<\omega\right\rangle$ be a sequence of Polish spaces and $\overline{\mathcal{I}}=\left\langle\mathcal{I}_{n}\right.$ : $n<\omega\rangle$ be a sequence of $\boldsymbol{\Pi}_{1}^{1}$ on $\boldsymbol{\Sigma}_{1}^{1} \sigma$-ideals generated by closed sets, $\mathcal{I}_{n}$ on $X_{n}$, respectively. If $A \subseteq \prod_{n<\omega} X_{n}$ is $\boldsymbol{\Sigma}_{1}^{1}$, then

- either $A \in \bigotimes_{n<\omega} \mathcal{I}_{n}$

- or $A$ contains an $\overline{\mathcal{I}}$-positive $\mathbf{G}_{\delta}$ cube $G$ such that

$$
\left(\Sigma_{1}^{1}\left(\prod_{n<\omega} X_{n}\right) \cap \bigotimes_{n<\omega} \mathcal{I}_{n}\right)\lceil G \subseteq \mathcal{M}(G) .
$$

Proof. Suppose $A \subseteq \prod_{n<\omega} X_{n}$ is an analytic $\bigotimes_{n<\omega} \mathcal{I}_{n}$-positive set. By Solecki's theorem and Theorem 1.1, the $\sigma$-ideals $\mathcal{I}_{n}$ are iterable, so we may apply Theorem 4.7 and assume that $A$ is an $\overline{\mathcal{I}}$-positive Bor cube. For each $n<\omega$ write $A_{n}$ for $\pi_{\omega, n}[A]$ and let $E_{n} \subseteq \prod_{i<n} X_{i} \times \omega^{\omega}$ be a closed set projecting to $A_{n}$. Let $\mathcal{G}_{n} \subseteq \omega^{\omega}$ be the analytic set from Lemma 3.6 consisting of codes of all $\mathcal{I}_{n}$-perfect $\mathbf{G}_{\delta}$ sets. In this proof we denote $\pi_{n, n-1}$ by $\pi_{n}$ and write $\overline{\mathcal{I}}_{n}$ for $\left\langle\mathcal{I}_{i}: i<n\right\rangle$.

We will use the following lemma.

Lemma 4.11 (Kuratowski, Ulam). Let $X$ and $Y$ be Polish spaces and let $f: Y \rightarrow$ $X$ be a continuous open surjection. Suppose $B \subseteq Y$ has the Baire property and

$$
\forall \mathcal{M}^{\mathcal{M}} \in X \quad B \cap f^{-1}[\{x\}] \text { is meager in } f^{-1}[\{x\}] .
$$

Then $B$ is meager in $Y$.

Proof. The proof is almost the same as the proof of the "product" version of the Kuratowski-Ulam theorem [9, Theorem 8.41]. The difference is that instead of [9, Lemma 8.42], we need to prove that if $U \subseteq Y$ is open dense, then

$$
\forall \forall^{\mathcal{M}} x \in X \quad U \cap f^{-1}[\{x\}] \text { is open dense in } f^{-1}[\{x\}] .
$$

To show this, we take the open basis $\left\langle U_{n}: n<\omega\right\rangle$ of $Y$ and show that for each $n<\omega$ the set

$$
V_{n}=\left\{x \in X: f^{-1}[\{x\}] \cap U_{n}=\emptyset \vee f^{-1}[\{x\}] \cap U_{n} \cap U \neq \emptyset\right\}
$$

contains an open dense set. Indeed, let $W_{n}=X \backslash \overline{f\left[U_{n}\right]}$ and notice that the set $f\left[U_{n}\right] \cup W_{n}$ is open dense in $X$. Moreover, $W_{n} \subseteq V_{n}$ and $V_{n} \cap f\left[U_{n}\right]$ is dense open in $f\left[U_{n}\right]$. Now, notice that if $x \in \bigcap_{n<\omega} V_{n}$, then $U \cap f^{-1}[\{x\}]$ is open dense in $f^{-1}[\{x\}]$. This proves $(*)$. 
We shall construct a sequence of $\mathbf{G}_{\delta}$ sets $G_{n} \subseteq \prod_{i<n} X_{i}$ such that

(i) $\pi_{n}\left[G_{n}\right] \subseteq G_{n-1}$ is comeager in $G_{n-1}$,

(ii) for each $x \in \pi_{n}\left[G_{n}\right]$ the set $\left(G_{n}\right)_{x}$ is $\mathcal{I}_{n}$-perfect,

(iii) $\pi_{n}\left\lceil G_{n}: G_{n} \rightarrow \pi_{n}\left[G_{n}\right]\right.$ is an open map,

(iv) if $D \subseteq \prod_{i<n} X_{i}$ is an $\overline{\mathcal{I}}_{n}$-full $\boldsymbol{\Sigma}_{1}^{1}$ cube, then $D \cap G_{n}$ is comeager in $G_{n}$.

Note that, by Lemma 4.5. (iv) implies

(v) $\left(\Sigma_{1}^{1}\left(\prod_{i<n} X_{i}\right) \cap \bigotimes_{i<n} \mathcal{I}_{i}\right)\left\lceil G_{n} \subseteq \mathcal{M}\left(G_{n}\right)\right.$.

For $n=0$ use Lemma 3.4 to find an $\mathcal{I}_{0}$-perfect $\mathbf{G}_{\delta}$-set $G_{0} \subseteq A_{0}$. Notice that $\left(\boldsymbol{\Sigma}_{1}^{1}\left(X_{0}\right) \cap \mathcal{I}_{0}\right)\left\lceil G_{0} \subseteq \mathcal{M}\left(G_{0}\right)\right.$ follows from the fact that $G_{0}$ is $\mathcal{I}_{0}$-perfect.

Suppose the set $G_{n} \subseteq X^{n}$ is constructed. Similarly as in Remark 4.4 we conclude that by Lemma 3.4, $\boldsymbol{\Sigma}_{2}^{1}$-absoluteness and continuous reading of names for $\mathbb{P}_{\mathcal{I}}$, for each $x \in G_{n}$ there is a code $c(x) \in \mathcal{G}_{n+1}$ for an $\mathcal{I}_{n+1}$-perfect $\mathbf{G}_{\delta}$ set $\tilde{G}_{c(x)}$ and a function $f: \tilde{G}_{c(x)} \stackrel{*}{\rightarrow}\left(E_{n+1}\right)_{x}$. Consider the set

$W=\left\{(x, d) \in X^{n} \times \omega^{\omega}: \quad x \in G_{n}, \exists c \in \mathcal{G}_{n+1} \exists f: \tilde{G}_{c} \stackrel{*}{\rightarrow}\left(E_{n+1}\right)_{x} \quad \operatorname{dom}(f)=\tilde{G}_{d}\right\}$.

$W$ is analytic and all vertical sections of $W$ are nonempty. Hence, by the Jankovvon Neumann theorem, $W$ has a $\sigma\left(\boldsymbol{\Sigma}_{1}^{1}\right)$-measurable uniformization $g: G_{n} \rightarrow \mathcal{G}_{n+1}$. In particular, $g$ is Baire measurable and hence it is continuous on a dense $\mathbf{G}_{\delta}$ set $G_{n}^{\prime} \subseteq G_{n}$. Let

$$
G_{n+1}=\left\{(x, y) \in X^{n} \times X: x \in G_{n}^{\prime} \wedge y \in \tilde{G}_{g(x)}\right\} .
$$

$G_{n+1}$ is a $\mathbf{G}_{\delta}$ set since $G_{n+1}=(g, i d)^{-1}[\tilde{G}]$. Moreover, $\pi_{n+1}\left[G_{n+1}\right]=G_{n}^{\prime}$ is comeager in $G_{n}$.

Note that the function $\pi_{n+1}\left\lceil G_{n+1}: G_{n+1} \rightarrow G_{n}^{\prime}\right.$ is open by Lemma 3.2 and the fact that $g$ is continuous on $G_{n}^{\prime}$.

Now, let $D \subseteq \prod_{i<n+1} X_{i}$ be an $\overline{\mathcal{I}}_{n+1}$-full $\boldsymbol{\Sigma}_{1}^{1}$ cube. The set $D_{n}=\pi_{n+1}[D]$ is an $\overline{\mathcal{I}}_{n}$-full $\Sigma_{1}^{1}$ cube, so, by the inductive hypothesis, $D_{n} \cap G_{n}$ is comeager in $G_{n}$. Therefore, $D_{n} \cap G_{n}^{\prime}$ is comeager in $G_{n}^{\prime}$. Moreover, if $x \in G_{n}^{\prime} \cap D_{n}$, then $D_{x} \cap\left(G_{n+1}\right)_{x}$ is comeager in $\left(G_{n+1}\right)_{x}$, since $\left(G_{n+1}\right)_{x}$ is $\mathcal{I}_{n+1}$-perfect. Now, $D$ has the Baire property, so by Lemma 4.11 (for the function $\pi_{n+1}\left\lceil G_{n+1}: G_{n+1} \rightarrow G_{n}^{\prime}\right.$ ) we have that $D \cap G_{n+1}$ is comeager in $G_{n+1}$.

This ends the construction.

Put

$$
G=\bigcap_{n<\omega} \pi_{\omega, n}^{-1}\left[G_{n}\right] .
$$

$G$ is a $\mathbf{G}_{\delta}$ set and it is contained in $A$ since $A$ is a $(\overline{\mathcal{I}}$-positive $)$ cube. For each $n, k<\omega$ also consider the set

$$
\begin{aligned}
& H_{n}^{k}=\left\{x_{n} \in G_{n}:\right. \forall \mathcal{M} y_{n+1} \in\left(G_{n+1}\right)_{x_{n}} \ldots \forall \forall^{\mathcal{M}} y_{n+k} \in\left(G_{n+k}\right)_{\left(x_{n}, y_{n+1}, \ldots, y_{n+k-1}\right)} \\
&\left.\exists y_{n+k+1} \in\left(G_{n+k+1}\right)_{\left(x_{n}, y_{n+1}, \ldots, y_{n+k}\right)} \quad\left(x_{n}, y_{n+1}, \ldots, y_{n+k+1}\right) \in G_{n+k}\right\} .
\end{aligned}
$$

Applying $(k+1)$-many times Lemma 4.11 we conclude that $H_{n}^{k}$ is comeager in $G_{n}$ for each $k<\omega$. Put

$$
H_{n}=\bigcap_{k<\omega} H_{n}^{k} .
$$


Each $H_{n}$ is also a comeager subset of $G_{n}$. Notice that $\pi_{n+1}\left[H_{n+1}\right] \subseteq H_{n}$ and $\pi_{n+1}\left[H_{n+1}\right]$ is comeager in $G_{n}$ for each $n<\omega$. Moreover, for each $n<m<\omega$

$$
\pi_{m, n}\left[H_{m}\right] \text { is comeager in } G_{n}
$$

(by repeatedly applying Lemma 4.11).

Notice that for each $n<\omega$ we have

$$
\bigcap_{n<m<\omega} \pi_{m, n}\left[H_{m}\right] \subseteq \pi_{\omega, n}[G] .
$$

Consequently, by $(*)$ we have that $\pi_{\omega, n}[G]$ is comeager in $G_{n}$. Therefore, it is $\bigotimes_{i<n} \mathcal{I}_{i}$-positive, by (v). For each $k<\omega$ and $x \in \pi_{\omega, k}[G]$ we may repeat the above argument in the space $\left(\prod_{n<\omega} X_{n}\right)_{x}$ and conclude that the set $\left(\pi_{\omega, k+1}[G]\right)_{x}$ is comeager in $\left(G_{k+1}\right)_{x}$ and hence is $\mathcal{I}_{k+1}$-positive (since $\left(G_{k+1}\right)_{x}$ is $\mathcal{I}_{k+1}$-perfect). Therefore $G$ is an $\overline{\mathcal{I}}$-positive $\mathbf{G}_{\delta}$ cube.

Now we prove that $\left(\boldsymbol{\Sigma}_{1}^{1}\left(\prod_{n<\omega} X_{n}\right) \cap \bigotimes_{n<\omega} \mathcal{I}_{n}\right)\lceil G \subseteq \mathcal{M}(G)$. By Theorem 4.8 it is enough to prove that if $D \subseteq \prod_{n<\omega} X_{n}$ is an $\overline{\mathcal{I}}$-full $\boldsymbol{\Sigma}_{1}^{1}$ cube, then $D \cap G$ is comeager in $G$. Write $D_{n}=\pi_{\omega, n}[D]$. Using (iv) we see that $D_{n}$ is comeager in $G_{n}$. For each $n<\omega$ find a dense in $G_{n} \mathbf{G}_{\delta}$ set $G_{n}^{\prime \prime} \subseteq D_{n}$ such that $G_{n+1}^{\prime \prime} \subseteq \pi_{n}^{-1}\left[G_{n}^{\prime \prime}\right]$. Let $G^{\prime \prime}=\bigcap_{n<\omega} \pi_{\omega, n}^{-1}\left[G_{n}^{\prime \prime}\right]$. Note that $G^{\prime \prime} \subseteq D \cap G$ and $G^{\prime \prime}$ is a $\mathbf{G}_{\delta}$ set. We will prove that $G^{\prime \prime}$ is dense in $G$.

Repeatedly applying Lemma 4.11 and property (iv) we see that for each $n<\omega$ the following holds:

$$
\forall^{\mathcal{M}} y_{0} \in G_{0} \forall^{\mathcal{M}} y_{1} \in\left(G_{1}\right)_{y_{0}} \ldots \forall^{\mathcal{M}} y_{n} \in\left(G_{n}\right)_{\left(y_{0}, \ldots, y_{n-1}\right)} \quad\left(y_{0}, \ldots, y_{n}\right) \in G_{n}^{\prime \prime} .
$$

Using this we can easily show $G^{\prime \prime}$ is nonempty, and, in fact, that if $U_{n} \subseteq X^{n}$ is open, then $G^{\prime \prime} \cap \pi_{\omega, n}^{-1}\left[U_{n}\right]$ is nonempty. But this implies that $G^{\prime \prime}$ is dense in $G$.

This ends the proof.

Let $X$ and $Y$ be Polish spaces and $F: X \rightarrow \mathcal{P}(Y)$ be a multifunction. If $\mathcal{A}$ is a family of subsets of $X$, then we say that $F$ is $\mathcal{A}$-measurable if for each open set $U \subseteq Y$ the set $F^{-1}(U)=\{x \in X: F(x) \cap U \neq \emptyset\}$ belongs to $\mathcal{A}$. We say that $F$ is an analytic multifunction if its graph, i.e. $\bigcup_{x \in X}\{x\} \times F(x)$, is analytic in $X \times Y$. The following result was motivated by the Kuratowski-Ryll Nardzewski theorem.

Proposition 4.12. Let $X$ be a Polish space and $\mathcal{I}$ a $\sigma$-ideal on $X$ generated by closed sets. If $F: X \rightarrow \mathcal{P}\left(\omega^{\omega}\right)$ is an analytic multifunction, then there is an I-positive $\mathbf{G}_{\delta}$ set $G$ such that $F\left\lceil G\right.$ is $\mathbf{\Sigma}_{3}^{0}$-measurable.

Proof. Denote the graph of $F$ by $A$ and let $a$ be such that $A \in \Sigma_{1}^{1}(a)$. Let $A(v, w)$ be a $\Sigma_{1}^{1}(a)$ formula defining the set $A$. Take $M \prec H_{\kappa}$ (for a large enough $\kappa$ ) containing $a$ and $\mathbb{P}_{\mathcal{I}}$. Let $G e n(M) \subseteq X$ be the set of all $\mathbb{P}_{\mathcal{I}}$-generic reals over $M$. $\operatorname{Gen}(M)$ is an $\mathcal{I}$-positive Borel set by properness of $\mathbb{P}_{\mathcal{I}}$. Find an $\mathcal{I}$-positive $\mathbf{G}_{\delta}$ set $G \subseteq \operatorname{Gen}(M)$. We will show that $F\left\lceil G\right.$ is $\boldsymbol{\Sigma}_{3}^{0}$-measurable. Notice that if $\tau \in \omega^{<\omega}$ and $x \in X$, then

$$
x \in F^{-1}([\tau]) \quad \text { iff } \quad \exists y \in[\tau] A(x, y) .
$$

This is a $\Sigma_{1}^{1}(a)$ formula, so it is absolute for $M[x] \subseteq V$. Therefore, by a usual forcing argument and the fact that $\mathcal{I}$-positive $\mathbf{G}_{\delta}$ sets are dense in $\mathbb{P}_{\mathcal{I}}$, we get

$$
F^{-1}[[\tau]]=\bigcup\left\{G \in \mathbb{P}_{\mathcal{I}} \cap M: \quad G \in \mathbf{G}_{\delta} \wedge G \Vdash \exists y \in[\tau] A(\dot{g}, y)\right\} .
$$

This is a $\boldsymbol{\Sigma}_{3}^{0}$ set. 


\section{FUSION GAMES}

In this section we briefly recall basic definitions concerning infinite games and introduce fusion games for $\sigma$-ideals.

By a game scheme we mean a set of rules for a two-player game (the players are called Adam and Eve, and Adam begins). Formally, a game scheme is a pruned tree (i.e. without terminal nodes) $G \subseteq Y^{<\omega}$ for a countable set $Y$ where the last elements of sequences at even and odd levels are understood as possible moves of Eve and Adam, respectively. In particular, in any game scheme the first move is made by Adam (the moves are numbered by $\omega \backslash\{0\}$ ). Nodes of the tree $G$ of even length are called partial plays and elements of $\lim (G)$ are called plays. Note that partial plays always end with a move of Eve.

If $\tau$ is a partial play in a game scheme $G$, then by the relativized game scheme $G_{\tau}$ we mean the tree $\left\{\sigma \in Y^{<\omega}: \tau^{\wedge} \sigma \in G\right\}$. The game scheme $G_{\tau}$ consists of the games which "continue" the partial play $\tau$.

A payoff set $p$ in a game scheme $G$ is a subset of $\lim (G)$. By a game we mean a pair $(G, p)$ where $G$ is a game scheme and $p$ is a payoff set in $G$ (we say that the game $(G, p)$ is in the game scheme $G)$. For a game $(G, p)$ we say that Eve wins a play $g \in \lim (G)$ if $g \in P$. Otherwise we say that Adam wins $g$.

A strategy for Adam in a game scheme $G$ is a subtree $S \subseteq G$ such that:

- for each odd $n \in \omega$ and $\tau \in S$ such that $|\tau|=n$, the set of immediate successors of $\tau$ in $S$ contains precisely one point,

- for each even $n \in \omega$ and $\tau \in S$ such that $|\tau|=n$, the sets of immediate successors of $\tau$ in $S$ and $G$ are equal.

The strategy for Eve is defined analogously. If $(G, p)$ is a game in the game scheme $G$ and $S$ is a strategy for Adam in $G$, then we say that $S$ is a winning strategy for Eve in the game $(G, p)$ if $\lim (S) \subseteq p$. Winning strategy for Adam is defined analogously.

Recall the classical Banach-Mazur game [9, Section 8.H] which "decides" whether a Borel set is meager or not, in terms of existence of a winning strategy for one of the players. Now we introduce an abstract notion of a fusion game which will cover the classical examples as well as those from Sections 7 and 8 Suppose we have a game scheme $G$ together with a family of payoff sets $p(A)$ for each $A \subseteq X$ such that:

(i) $p(A) \subseteq \lim (G)$ is Borel, for each Borel set $A \subseteq X$,

(ii) $p(A) \subseteq p(B)$ for each $B \subseteq A$,

(iii) $p\left(\bigcup_{n<\omega} A_{n}\right)=\bigcap_{n<\omega} p\left(\overline{A_{n}}\right)$ for each sequence $\left\langle A_{n}: n<\omega\right\rangle$.

Intuitively, $p(A)$ is such that a winning strategy for Eve in $(G, p(A))$ "proves" that $A$ is "small". For each $A \subseteq X$ the game $G(A)$ is the game in the game scheme $G$ with the payoff set $p(A)$. We denote by $G(\cdot)$ the game scheme $G$ together with the function $p$. We call $G(\cdot)$ a fusion scheme if

(iv) the moves of Adam code (in a prescribed way, in terms of a fixed enumeration of the basis) basic open sets $U_{n}$ such that $\overline{U_{n+1}} \subseteq U_{n}$ and $\operatorname{diam}\left(U_{n}\right)<1 / n$,

(v) for each Borel set $A \subseteq X$ and each play $g$ in $G(A)$ if Adam wins $g$, then the single point in the intersection of $U_{n}$ 's (as above) is in $A$. 
Notice that if the family of sets $q \subseteq \lim (G)$ such that Eve has a winning strategy in the game $(G, q)$ is closed under countable intersections, then the family of sets $A \subseteq X$ such that Eve has a winning strategy in $G(A)$ forms a $\sigma$-ideal (by (iii)).

The idea of considering $\sigma$-ideals defined in terms of a winning strategy in a game scheme occurs in a paper of Schmidt [17] and later in a work of Mycielski [14.

If the family of sets $A \subseteq X$ for which Eve has a winning strategy in $G(A)$ forms a $\sigma$-ideal $\mathcal{I}$, then we say that $G(\cdot)$ is a fusion scheme for $\mathcal{I}$.

Suppose $X=\lim (T)$ for some countable tree $T$. Also suppose that the game scheme $G$ is such that the possible $n$-th moves of Adam correspond to elements at the $n$-th level of $T$ (such as in (iv), to the basic clopen sets $[\tau]_{T}$ for $\tau \in \operatorname{Lev}_{n}(T)$ ). Let $G_{\mathcal{I}}(\cdot)$ be a fusion scheme for a $\sigma$-ideal $\mathcal{I}$ and let $\tau$ be a partial play in the game scheme $G_{\mathcal{I}}$. Let $U$ be the basic clopen set coded by the last move of Adam in $\tau$. Recall that the relativized game scheme $\left(G_{\mathcal{I}}\right)_{\tau}$ consists of the continuations of $\tau$ in $G_{\mathcal{I}}$. The game scheme $\left(G_{\mathcal{I}}\right)_{\tau}$, together with the function $p_{\tau}(A)=p(A) \cap U$, defines a relativized fusion scheme. Using the property $(\mathrm{v})$ we easily get the following.

Proposition 5.1. Let $X=\lim (T), G_{\mathcal{I}}, \tau$ and $U$ be as above. Let $A \subseteq X$. Eve has a winning strategy in $\left(G_{\mathcal{I}}\right)_{\tau}(A)$ if and only if Eve has a winning strategy in $G_{\mathcal{I}}(A \cap U)$.

\section{FUSION IN THE FORCING $\mathbb{P}_{\mathcal{I}}$}

In this section we give a proof of Theorem 1.3 . The main ingredient here is fusion schemes for $\sigma$-ideals generated by closed sets.

We now give an informal outline of the proof of Theorem 1.3. The general idea is as follows: having a $\sigma$-ideal $\mathcal{I}$ generated by closed sets, we find a fusion scheme $G_{\mathcal{I}}(\cdot)$ for $\mathcal{I}$ such that the trees of winning strategies in $G_{\mathcal{I}}(B)$ (for $B \in \mathbb{P}_{\mathcal{I}}$ ) determine some analytic $\mathcal{I}$-positive sets. Moreover, for each $B \in \mathbb{P}_{\mathcal{I}}$ the winning condition for Adam (the complement of the payoff set) in $G_{\mathcal{I}}(B)$ is a $\mathbf{G}_{\delta}$ set in $\lim \left(G_{\mathcal{I}}\right)$. We consider the forcing with trees of winning strategies for Adam in the games $G_{\mathcal{I}}(B)$ (for all $B \in \mathbb{P}_{\mathcal{I}}$ ) and show that it is equivalent to the original forcing $\mathbb{P}_{\mathcal{I}}$ (we in fact show that it is equivalent to the forcing with $\mathcal{I}$-positive $\boldsymbol{\Sigma}_{1}^{1}$ sets and then use Solecki's theorem to conclude that all three forcings are equivalent). Using the fact that the winning conditions in $G_{\mathcal{I}}(B)$ are the intersections of $\omega$ many open sets, we define $\omega$ many fronts in the trees of winning strategies (such that crossing the $n$-th front implies that the game is in the $n$-th open set). Now, using these fronts as analogues of the splitting levels in perfect or superperfect trees, we define fusion in the forcing of winning strategies for Adam.

Although the general idea is based on the above outline, we will have to additionally modify the games in order to avoid some determinacy problems. That is, instead of a fusion scheme for $\mathcal{I}$ and the games $G_{\mathcal{I}}(B)$ we will use their unfolded variant. We would like to emphasize that in many concrete cases of $\sigma$-ideals (such as in Sections 7 or 8), we can use simpler fusion schemes and the fusion from Theorem 1.3 can be simplified.

Proof of Theorem 1.3. To simplify notation we assume that the underlying space $X$ is the Baire space $\omega^{\omega}$. Pick a bijection $\rho: \omega \rightarrow \omega \times \omega$. $H_{\omega_{1}}$ stands for the family of hereditarily countable sets (it will be used to make sure that the forcing we define is a set). 
We will use the following notation:

- Let $Y$ be an arbitrary set. If $\tau \in(\omega \times Y)^{<\omega}$, then by $\bar{\tau} \in \omega^{<\omega}$ we denote the sequence of the first coordinates of the elements of $\tau$. Suppose $T$ is a tree on $\omega \times Y$. The map p $\mathrm{p}_{Y}: \lim (T) \rightarrow \omega^{\omega}$ is defined as follows: if $t \in \lim (T)$ and $t\left\lceil n=\tau_{n}\right.$, then $\mathrm{p}_{Y}(t)=\bigcup_{n<\omega} \bar{\tau}_{n}$.

- Let $z$ be arbitrary. If $\tau \in(\omega \times Y)^{<\omega}$ and $\tau=\left\langle a_{i}: i<|\tau|\right\rangle$, then by $\tau^{z} \in(\omega \times Y \times\{z\})^{<\omega}$ we denote the sequence $\left\langle\left(a_{i}, z\right): i<|\tau|\right\rangle$. If $T$ is a tree on $\omega \times Y$, then by $T^{z}$ we denote the tree $\left\{\tau^{z}: \tau \in T\right\}$ on $\omega \times Y \times\{z\}$. - If $Y=W \times Z$ and $\tau \in(\omega \times Y)^{<\omega}$, then by $\tau_{W} \in(\omega \times W)^{<\omega}$ we denote $\left\langle\pi_{\omega \times W}\left(a_{i}\right): i<|\tau|\right\rangle$ (where $\pi_{\omega \times W}: \omega \times W \times Z \rightarrow \omega \times W$ is the projection to the first two coordinates). By $T_{W}$ we denote the tree $\left\{\tau_{W}: \tau \in T\right\}$.

For $Y \in H_{\omega_{1}}$ and a tree $T$ on $\omega \times Y$ let $G_{\mathcal{I}}(Y, T)$ be the game scheme in which

- in his $n$-th turn Adam constructs $\tau_{n} \in T$ such that $\tau_{n} \supsetneq \tau_{n-1}\left(\tau_{-1}=\emptyset\right)$,

- in her $n$-th turn Eve picks a clopen set $O_{n}$ in $\omega^{\omega}$ such that

$$
\operatorname{proj}\left[T\left(\tau_{n}\right)\right] \notin \mathcal{I} \Rightarrow O_{n} \cap \operatorname{proj}\left[T\left(\tau_{n}\right)\right] \notin \mathcal{I} .
$$

By the end of a play, Adam and Eve have a sequence of closed sets $E_{k}$ in $\omega^{\omega}$ defined as follows:

$$
E_{k}=2^{\omega} \backslash \bigcup_{i<\omega} O_{\rho^{-1}(i, k)} .
$$

Put $x=\bigcup_{n<\omega} \bar{\tau}_{n} \in \omega^{\omega}$. Consider a payoff set in $G_{\mathcal{I}}(Y, T)$ such that Adam wins if and only if

$$
x \notin \bigcup_{k<\omega} E_{k} .
$$

In this proof, the game in the game scheme $G_{\mathcal{I}}(Y, T)$ with the above payoff set will also be denoted by $G_{\mathcal{I}}(Y, T)$ (this should not cause confusion since we are not going to consider other payoff sets in the game scheme $G_{\mathcal{I}}(Y, T)$ ).

Here is more notation:

- If $S$ is a subtree of the game scheme $G_{\mathcal{I}}(Y, T)$, then by $\hat{S} \subseteq T$ we denote the tree built from the moves of Adam in partial plays in $S$ (i.e. we forget about Eve's moves). We write $\operatorname{proj}[S]$ for $\operatorname{proj}[\hat{S}]$.

- If $Y^{\prime}=Y \times Z, z \in Z$ is fixed and $T^{\prime}$ is a tree on $Y^{\prime}$ such that $T^{z} \subseteq T$, then by $S^{z}$ we denote the subtree of $G_{\mathcal{I}}\left(Y^{\prime}, T^{\prime}\right)$, in which the moves $\tau$ of Adam are changed to $\tau^{z}$.

Lemma 6.1. The game $G_{\mathcal{I}}(Y, T)$ is determined. Eve has a winning strategy in $G_{\mathcal{I}}(Y, T)$ if and only if

$$
\operatorname{proj}[T] \in \mathcal{I} .
$$

Proof. First suppose that $\operatorname{proj}[T] \in \mathcal{I}$. Then Eve chooses $\emptyset$ in all her moves and wins the game.

On the other hand, suppose that $\operatorname{proj}[T]$ is $\mathcal{I}$-positive. We define a winning strategy for Adam as follows. In his moves, Adam constructs $\tau_{n} \in T$ so that

- $\left[\bar{\tau}_{n}\right] \subseteq O_{n-1}$,

- $\operatorname{proj}\left[T\left(\tau_{n}\right)\right] \notin \mathcal{I}$.

Suppose Adam is about to make his $n$-th move, his previous move is $\tau_{n-1}$ and the last move of Eve is $O_{n-1}\left(O_{-1}=\emptyset\right)$. Using the fact that $O_{n-1} \cap \operatorname{proj}\left[T\left(\tau_{n-1}\right)\right] \notin \mathcal{I}$, Adam picks $\tau_{n} \in T$ extending $\tau_{n-1}$ such that $\left[\bar{\tau}_{n}\right] \subseteq O_{n-1}$ and $\operatorname{proj}\left[T\left(\tau_{n}\right)\right] \notin \mathcal{I}$. 
This is the strategy for Adam. It is winning since after each play we have that $x \in O_{n}$ for each $n<\omega$, so in particular $x \in \bigcup_{k<\omega} E_{k}$.

Remark 6.2. Note that if $S$ is a winning strategy for Adam in the game $G_{\mathcal{I}}(Y, T)$, then for each partial play $\pi \in S$, we have $\operatorname{proj}[S(\pi)] \notin \mathcal{I}$. This is because otherwise we could construct a counterplay to the strategy $S$. In particular, if $\tau$ is the last move of Adam in $\pi$, then we have $\operatorname{proj}[T(\tau)] \notin \mathcal{I}$.

Now we define the key notion in this proof. Let $\pi$ be a partial play in $G_{\mathcal{I}}(Y, T)$ of length $2 l$, in which Eve chooses clopen sets $O_{i}$, for $i<l$, and Adam picks $\tau_{l-1} \in T$ in his last move. Suppose that $Y^{\prime}=Y \times Z, Z \in H_{\omega_{1}}, z \in Z$ is fixed and $T^{\prime}$ is a tree on $\omega \times Y^{\prime}$ such that $\left(\tau_{l-1}\right)^{z} \in T^{\prime}$. By the relativized unfolded game $G_{\mathcal{I}}\left(Y^{\prime}, T^{\prime}\right)_{\pi}^{z}$ we mean the game in which

- in his $n$-th move Adam picks $\tau_{n+l}^{\prime} \in T^{\prime}, \tau_{n+l}^{\prime} \supsetneq \tau_{n+l-1}^{\prime}\left(\tau_{l-1}^{\prime}=\left(\tau_{l-1}\right)^{z}\right)$,

- in her $n$-th move Eve picks a clopen set $O_{n+l}$ in $\omega^{\omega}$ such that

$$
\operatorname{proj}\left[T^{\prime}\left(\tau_{n+l}^{\prime}\right)\right] \notin \mathcal{I} \Rightarrow O_{n+l} \cap \operatorname{proj}\left[T^{\prime}\left(\tau_{n+l}^{\prime}\right)\right] \notin \mathcal{I} .
$$

The payoff set is the same as in the unrelativized case, i.e. we use all $\left\langle O_{n}: n<\omega\right\rangle$ to define a sequence of closed sets $\left\langle E_{k}: k<\omega\right\rangle$, we put $x=\bigcup_{l-1 \leq n<\omega} \overline{\tau_{n}^{\prime}}$ and Eve wins if and only if $x \in \bigcup_{k<\omega} E_{k}$.

With an analogous proof as in Lemma 6.1 we get the following lemma.

Lemma 6.3. Suppose $\pi$ is a partial play in $G_{\mathcal{I}}(Y, T)$ and $\tau$ is the last move of Adam in $\pi$. Let $G_{\mathcal{I}}\left(Y^{\prime}, T^{\prime}\right)_{\pi}^{z}$ be a relativized unfolded game. Eve has a winning strategy in $G_{\mathcal{I}}\left(Y^{\prime}, T^{\prime}\right)_{\pi}^{z}$ if and only if

$$
\operatorname{proj}\left[T^{\prime}\left(\tau^{z}\right)\right] \in \mathcal{I} \text {. }
$$

Lemma 6.4. If $S$ is a winning strategy for Adam in $G_{\mathcal{I}}(Y, T)$, then

$$
\operatorname{proj}[S] \notin \mathcal{I} \text {. }
$$

Proof. Let $A=\operatorname{proj}[S]$. If $A \in \mathcal{I}$, then there are closed sets $E_{k} \in \mathcal{I}$ such that $A \subseteq \bigcup_{k<\omega} E_{k}$. Let $U_{k}^{m}$ be clopen sets such that $U_{k}^{m} \subseteq U_{k}^{m+1}$ and $\omega^{\omega} \backslash E_{k}=$ $\bigcup_{m<\omega} U_{k}^{m}$ for each $k<\omega$. We construct an Eve's counterplay to the strategy $S$ in the following way. Suppose she is to make her $n$-th move and let $\tau_{n}$ be the last move of Adam. By Remark [6.2, $\operatorname{proj}\left[T\left(\tau_{n}\right)\right] \notin \mathcal{I}$. Let $\rho(n)=(i, k)$. She chooses $m \geq n$ large enough so that

$$
U_{k}^{m} \cap \operatorname{proj}\left[T\left(\tau_{n}\right)\right] \notin \mathcal{I} .
$$

Let her $n$-th move be $O_{n}=U_{k}^{m}$. If she plays in this way, then

$$
\bigcup_{i<\omega} O_{\rho^{-1}(i, k)}=\omega^{\omega} \backslash E_{k},
$$

i.e., the closed sets she gets are precisely the sets $E_{k}$. If $x=\bigcup_{n<\omega} \bar{\tau}_{n}$ is the point in $\omega^{\omega}$ constructed by Adam, then by the definition of $A, x \in A \subseteq \bigcup_{k<\omega} E_{k}$, which shows that Eve wins.

Note that it follows from Lemmas 6.1 and 6.4 that any analytic $\mathcal{I}$-positive set $A \subseteq \omega^{\omega}$ contains an analytic $\mathcal{I}$-positive subset of the form proj $[S]$ for a winning strategy $S$ for Adam in a game $G_{\mathcal{I}}(Y, T)$ (where $Y=\omega$ and $T$ is a tree on $\omega \times \omega$ such that $A=\operatorname{proj}[T])$. 
Let $\mathbb{T}_{\mathcal{I}}$ be the set of all triples $(Y, T, S)$ where $Y \in H_{\omega_{1}}, T$ is a tree on $\omega \times Y$ and $S$ is a winning strategy for Adam in the game $G_{\mathcal{I}}(Y, T)$. $\mathbb{T}_{\mathcal{I}}$ is a forcing notion with the following ordering: for $\left(Y^{\prime}, T^{\prime}, S^{\prime}\right),(Y, T, S) \in \mathbb{T}_{\mathcal{I}}$ let

$$
\left(Y^{\prime}, T^{\prime}, S^{\prime}\right) \leq(Y, T, S) \quad \text { iff } \operatorname{proj}\left[S^{\prime}\right] \subseteq \operatorname{proj}[S] .
$$

Notice that $(Y, T, S) \mapsto \operatorname{proj}[S]$ is a dense embedding from $\mathbb{T}_{\mathcal{I}}$ to $\mathbb{Q}_{\mathcal{I}}=\left(\boldsymbol{\Sigma}_{1}^{1} \backslash\right.$ $\mathcal{I}, \subseteq)$. Indeed, suppose that $\left(Y^{\prime}, T^{\prime}, S^{\prime}\right) \perp(Y, T, S)$. If $\operatorname{proj}\left[S^{\prime}\right]$ and $\operatorname{proj}[S]$ were compatible in $\mathbb{Q}_{\mathcal{I}}$, then we would find an $\mathcal{I}$-positive $\Sigma_{1}^{1}$ set $A \subseteq \omega^{\omega}$ such that $A \subseteq \operatorname{proj}\left[S^{\prime}\right] \cap \operatorname{proj}[S]$. Take any tree $T$ on $\omega \times \omega$ such that $\operatorname{proj}[T]=A$ and find a winning strategy $S^{\prime \prime}$ for Adam in $G_{\mathcal{I}}(\omega, T)$. Then $\left(\omega, T, S^{\prime \prime}\right) \leq\left(Y^{\prime}, T^{\prime}, S^{\prime}\right),(Y, T, S)$, a contradiction.

By Solecki's theorem, $\mathbb{P}_{\mathcal{I}}$ is dense in $\mathbb{Q}_{\mathcal{I}}$. Therefore the three forcing notions $\mathbb{T}_{\mathcal{I}}, \mathbb{Q}_{\mathcal{I}}$ and $\mathbb{P}_{\mathcal{I}}$ are equivalent. We will show that the forcing $\mathbb{T}_{\mathcal{I}}$ satisfies Axiom A.

Take $(Y, T, S) \in \mathbb{T}_{\mathcal{I}}$ and recall that for each play $p \in \lim (S)$ ending with $t \in$ $\omega^{\omega} \times Y^{\omega}$, with $x \in \omega^{\omega}$ (defined from the moves of Adam) and a sequence of closed sets $E_{n}$ (defined from the moves of Eve), we have $x \notin \bigcup_{k} E_{k}$. Note that for each $k \in \omega$ there is $n \in \omega$ (even) such that (the partial play) $t\lceil n$ already determines that $x \notin E_{k}$ (i.e. $\left[\bar{\tau}_{n}\right] \subseteq O_{m}$ for some $m<\omega$ such that $\rho(m)=(i, k)$ for some $i<\omega$ ). Let $n_{0}(p) \in \omega$ be the minimal such $n$ for $k=0$. Put

$$
F_{0}(S)=\left\{p\left\lceil n_{0}(p): p \in \lim (S)\right\} .\right.
$$

Note that $F_{0}(S)$ is a front in $S$. Analogously we define $F_{k}(S)$ for each $k<\omega$ (instead of $E_{0}$ take $E_{k}$ and put $n_{k}(p)>n_{k-1}(p)$ as a minimal even number such that $p\left\lceil n_{k}(p)\right.$ determines $\left.x \notin E_{k}\right)$.

Define $\left(Y^{\prime}, T^{\prime}, S^{\prime}\right) \leq_{k}(Y, T, S)$ iff

(i) $\left(Y^{\prime}, T^{\prime}, S^{\prime}\right) \leq(Y, T, S)$,

(ii) there is $Z \in H_{\omega_{1}}$ such that $Y^{\prime}=Y \times Z$,

(iii) there is $z \in Z$ such that $T^{z} \subseteq T^{\prime}$,

(iv) $\left(T^{\prime}\right)_{Y} \subseteq \hat{S}$,

(v) $F_{k}\left(S^{\prime}\right)=F_{k}(S)^{z}$.

We will prove that $\mathbb{T}_{\mathcal{I}}$ satisfies Axiom A with the inequalities $\leq_{k}$. Condition (ii) serves for unfolding the game and condition (iii) is used later to make the unfolding "rigid". Condition (iv) is a technical detail. The crucial one is (v), which says that the "splitting levels" are kept up to $k$-th in the $k$-th step of the fusion.

1. Fix $k<\omega$. Suppose that $(Y, T, S) \in \mathbb{T}_{\mathcal{I}}$ and $\dot{\alpha}$ is a name for an ordinal. We shall find $\left(Y^{\prime}, T^{\prime}, S^{\prime}\right) \leq_{k}(Y, T, S)$ and a countable set of ordinals $A$ such that $\left(Y^{\prime}, T^{\prime}, S^{\prime}\right) \Vdash_{\mathbb{T}_{\mathcal{I}}} \dot{\alpha} \in \check{A}$.

For each $\pi \in F_{k}(S)$ find an ordinal $\alpha_{\pi}$ and an $\mathcal{I}$-positive analytic set $A_{\pi} \subseteq$ $\operatorname{proj}[S(\pi)]$ (recall that $\operatorname{proj}[S(\pi)]$ is $\mathcal{I}$-positive by Remark 6.2) such that

$$
A_{\pi} \Vdash_{\mathbb{Q}_{\mathcal{I}}} \dot{\alpha}=\check{\alpha}_{\pi} .
$$

Let $\tau_{\pi} \in T$ be the last move of Adam in $\pi$. Next, pick $Z_{\pi} \in H_{\omega_{1}}$ such that $0 \in Z_{\pi}$ (0 will be used as $z$ from (iii); it does not matter what element we choose for $z$, as long as it belongs to $\left.H_{\omega_{1}}\right)$ and find a pruned tree $T_{\pi}$ on $\omega \times Y \times Z_{\pi}$ such that

- $\tau_{\pi}^{0} \in T_{\pi}$ and $\tau_{\pi}^{0}$ is a stem of $T_{\pi}$,

- $\left(T_{\pi}\right)_{Y} \subseteq \hat{S}$

- $A_{\pi}=\operatorname{proj}\left[T_{\pi}\right]$. 
( $T_{\pi}$ is chosen such that its projection to $\omega^{\omega} \times Y^{\omega}$ is the analytic set $\lim (\hat{S}) \cap \mathrm{p}_{Y}^{-1}\left[A_{\pi}\right]$.) Ensure also that

- for each $\pi, \pi^{\prime} \in F_{k}(S)$, if $\pi \neq \pi^{\prime}$, then $Z_{\pi} \cap Z_{\pi^{\prime}}=\{0\}$,

- for each $\tau \in T_{\pi}$ if $\tau \supsetneq\left(\tau_{\pi}\right)^{0}$, then $\tau(|\tau|-1) \in \omega \times Y \times\left(Z_{\pi} \backslash\{0\}\right)$.

Put

$$
Z=\bigcup_{\pi \in F_{k}(S)} Z_{\pi}, \quad z=0, \quad Y^{\prime}=Y \times Z .
$$

For each $\pi \in F_{k}(S)$ let $S_{\pi}$ be a winning strategy for Adam in $G_{\mathcal{I}}\left(Y \times Z_{\pi}, T_{\pi}\right)_{\pi}^{0}$. Such a strategy exists by Lemma 6.3 since $\operatorname{proj}\left[T_{\pi}\left(\tau_{\pi}{ }^{0}\right)\right]=\operatorname{proj}\left[T_{\pi}\right]=A_{\pi} \notin \mathcal{I}$ (recall that $\tau_{\pi}^{0}$ is a stem of $T_{\pi}$ ). Let

$$
T^{\prime}=T^{0} \cup \bigcup_{\pi \in F_{k}(S)} T_{\pi}
$$

and consider the game $G_{\mathcal{I}}\left(Y^{\prime}, T^{\prime}\right)$. The tree

$$
S^{\prime}=\bigcup_{\pi \in F_{n}(S)}\left(\pi^{0}\right)^{\frown} S_{\pi}
$$

is a strategy in $G\left(Y^{\prime}, T^{\prime}\right)$ since all $\pi^{0}$, for $\pi \in F_{k}(S)$, are partial plays in $G\left(Y^{\prime}, T^{\prime}\right)$ (because $T^{0} \subseteq T^{\prime}$ ). Moreover, it is a winning strategy for Adam since each $S_{\pi}$ is a winning strategy for Adam in $G_{\mathcal{I}}\left(Y \times Y_{\pi}, T_{\pi}\right)_{\pi}$. Therefore $\left(Y^{\prime}, T^{\prime}, S^{\prime}\right) \in \mathbb{T}_{\mathcal{I}}$. By the construction we have $\left(Y^{\prime}, T^{\prime}, S^{\prime}\right) \leq_{k}(Y, T, S)$. Moreover,

$$
\left(Y^{\prime}, T^{\prime}, S^{\prime}\right) \Vdash_{\mathbb{T}_{\mathcal{I}}} \dot{\alpha} \in\left\{\alpha_{\tau}: \tau \in F_{k}(S)\right\}
$$

because the set $\left\{\operatorname{proj}\left[S^{\prime}(\pi)\right]: \pi \in F_{k}(S)\right\}$ is predense below proj $\left[S^{\prime}\right]$ and we have $\operatorname{proj}\left[S^{\prime}(\pi)\right] \Vdash_{\mathbb{Q}_{\mathcal{I}}} \dot{\alpha}=\check{\alpha}_{\pi}$ (since $\left.\operatorname{proj}\left[S^{\prime}(\pi)\right] \subseteq A_{\pi}\right)$.

2. Let $\left\langle\left(Y_{k}, T_{k}, S_{k}\right): k<\omega\right\rangle$ be a fusion sequence. For each $k<\omega$ let $Z_{k} \in H_{\omega_{1}}$ be such that $Y_{k+1}=Y_{k} \times Z_{k}$ and let $z_{k} \in Z_{k}$ be as in the definition of $\leq_{k}$. Let $\vec{z}_{k}=\left\langle z_{k}, z_{k+1}, \ldots\right\rangle$. Put $Y=\bigcup_{k<\omega}\left(Y_{k}\right)^{\vec{z}_{k}}$ and $T=\bigcup_{k<\omega}\left(T_{k}\right)^{\vec{z}_{k}}$. $T$ is a tree on $Y$. Notice that for each $k<\omega$, for each $\tau \in T_{k}$ we have

$$
\operatorname{proj}\left[T_{k}(\tau)\right]=\operatorname{proj}\left[T\left(\tau^{\vec{z}_{k}}\right)\right] \text {. }
$$

Indeed, $\operatorname{proj}\left[T_{k}(\tau)\right] \subseteq \operatorname{proj}\left[T\left(\tau^{\vec{z}_{k}}\right)\right]$ follows from (iii) and $\operatorname{proj}\left[T\left(\tau^{\vec{z}_{k}}\right)\right] \subseteq \operatorname{proj}\left[T_{k}(\tau)\right]$ from (iv) (because for each $t \in \lim \left(T\left(\tau^{\vec{z}_{k}}\right)\right.$ ) a sequence of its initial coordinates is in $\lim \left(\hat{S}_{k}\right)$ and hence in $\left.\lim \left(T_{k}\right)\right)$.

Consider the game $G_{\mathcal{I}}(Y, T)$ and let

$$
S=\bigcup_{k<\omega} F_{k}\left(S_{k}\right)^{\vec{z}_{k}} .
$$

Note that it follows from (*) that $S$ is a strategy for Adam in $G_{\mathcal{I}}(Y, T)$. Moreover, for each $k<\omega$ we have $F_{k}(S)=F_{k}\left(S_{k}\right)^{\vec{z}_{k}}$, by the definition of $F_{k}$. Since for each $p \in \lim (S)$ we have

$$
\forall k<\omega \exists m<\omega \quad p\left\lceil m \in F_{k}(S),\right.
$$

it follows that $S$ is a winning strategy for Adam in $G_{\mathcal{I}}(Y, T)$. Therefore $(Y, T, S) \in$ $\mathbb{T}_{\mathcal{I}}$.

To see that $(Y, T, S) \leq\left(Y_{k}, T_{k}, S_{k}\right)$ we use property (iv). Indeed, if $x \in \operatorname{proj}[S]$, then there is a play in $\lim (S)$ in which $x$ is defined. By (iv), however, we can extract from this play a play in $\lim \left(S_{k}\right)$ in which $x$ is defined.

To check that $(Y, T, S) \leq_{k}\left(Y_{k}, T_{k}, S_{k}\right)$ we put $Z=\prod_{m \geq k} Z_{m}$ and $z=\vec{z}_{k}$.

This ends the proof of Theorem 1.3. 


\section{Closed null sets}

We denote by $\mathcal{E}$ the $\sigma$-ideal generated by closed null sets in $2^{\omega}$ (with respect to the standard Haar measure $\mu$ on $2^{\omega}$ ). The sets in $\mathcal{E}$ are both null and meager. $\mathcal{E}$ is properly contained in $\mathcal{M} \cap \mathcal{N}$ [2, Lemma 2.6.1] and, in fact, one can show that $\mathcal{E}$ is not ccc.

The family of closed sets in $\mathcal{E}$ coincides with the family of closed null sets and $\mathcal{E} \cap \boldsymbol{\Pi}_{1}^{0}\left(2^{\omega}\right)$ is a $\mathbf{G}_{\delta}$ set in $K\left(2^{\omega}\right)$ (for each $\varepsilon>0$ the set $\left\{C \in K\left(2^{\omega}\right): \mu(C)<\varepsilon\right\}$ is open). Therefore, $\mathcal{E}$ is $\boldsymbol{\Pi}_{1}^{1}$ on $\boldsymbol{\Sigma}_{1}^{1}$ by Corollary 3.8 .

The forcing $\mathbb{P}_{\mathcal{E}}$ adds an unbounded real and a splitting real. In fact, one can check that the generic real is splitting. To see that $\mathbb{P}_{\mathcal{E}}$ adds an unbounded real, recall a theorem of Zapletal [22, Theorem 3.3.2], which says that a forcing $\mathbb{P}_{\mathcal{I}}$ is $\omega^{\omega}$-bounding if and only $\mathbb{P}_{\mathcal{I}}$ has continuous reading of names and compact sets are dense in $\mathbb{P}_{\mathcal{I}}$. Let $G$ be a $\mathbf{G}_{\delta}$ set such that $G \in \mathcal{N}$ and $2^{\omega} \backslash G \in \mathcal{M}$. $G$ is $\mathcal{E}$-positive, but no compact $\mathcal{E}$-positive set is contained in $G$. In particular, compact sets are not dense in $\mathbb{P}_{\mathcal{E}}$ and hence this forcing is not $\omega^{\omega}$-bounding. $\mathbb{P}_{\mathcal{E}}$ does not, however, add a dominating real. This follows from another theorem of Zapletal [22, Theorem 3.8.15], which says that if $\mathcal{I}$ is a $\boldsymbol{\Pi}_{1}^{1}$ on $\boldsymbol{\Sigma}_{1}^{1} \sigma$-ideal, then the forcing $\mathbb{P}_{\mathcal{I}}$ does not add a dominating real.

Zapletal proved in [22, Theorem 4.1.7] (see the first paragraph of the proof) that if $\mathcal{I}$ is a $\sigma$-ideal generated by an analytic collection of closed sets and $V \subseteq V[G]$ is a $\mathbb{P}_{\mathcal{I}}$-extension, then any intermediate extension $V \subseteq W \subseteq V[G]$ is equal either to $V$ or $V[G]$, or is an extension by a Cohen real. Therefore it is natural to ask if $\mathbb{P}_{\mathcal{E}}$ adds Cohen reals.

Recall that a closed set $D \subseteq 2^{\omega}$ is self-supporting if for any clopen set $U$ the set $D \cap U$ is either empty or not null. Notice that a closed set is self-supporting if and only if it is $\mathcal{E}$-perfect. If $\mu$ is a Borel measure on $X$ and $A \in \operatorname{Bor}(X)$ is such that $\mu(A)>0$, then by $\mu_{A}$ we denote the relative measure on $A$ defined as $\mu_{A}(B)=\mu(A \cap B) / \mu(A)$.

Theorem 7.1. The forcing $\mathbb{P}_{\mathcal{E}}$ does not add Cohen reals.

Proof. Suppose $B \in \mathbb{P}_{\mathcal{E}}$ and $\dot{x}$ is a name for a real such that

$$
B \Vdash \dot{x} \text { is a Cohen real. }
$$

By Lemma 3.4 and continuous reading of names we find a $\mathbf{G}_{\delta}$ set $G \subseteq B$ such that $D=\bar{G}$ is self-supporting and a continuous function $f: G \rightarrow \omega^{\omega}$ such that $G \Vdash \dot{x}=f(\dot{g})$. Pick a continuous, strictly positive measure $\nu$ on $G$. For each $\tau \in \omega^{<\omega}$ the set $C_{\tau}=f^{-1}[[\tau]]$ is a relative clopen set in $G$. Find open sets $C_{\tau}^{\prime} \subseteq D$ such that $C_{\tau}=C_{\tau}^{\prime} \cap G$. $D$ is zero-dimensional, so by the reduction property for open sets we may assume that

- $C_{\tau_{0}}^{\prime} \subseteq C_{\tau_{1}}^{\prime}$ for $\tau_{0} \subseteq \tau_{1}$,

- $C_{\tau_{0}}^{\prime} \cap C_{\tau_{1}}^{\prime}=\emptyset$ for $\tau_{0} \perp \tau_{1}$.

We will find a tree $T \subseteq \omega^{<\omega}$ such that $\lim (T)$ is nowhere dense in $\omega^{\omega}$ and the closure of the set $f^{-1}[\lim (T)]$ is self-supporting.

Enumerate all nonempty clopen sets in $D$ in a sequence $\left\langle V_{n}^{\prime}: n<\omega\right\rangle$ and all nonempty clopen sets in $G$ in a sequence $\left\langle V_{n}: n<\omega\right\rangle$, and elements of $\omega<\omega$ in a sequence $\left\langle\sigma_{n}: n<\omega\right\rangle$. If $\tau \in \omega^{<\omega}$, then $\left\langle C_{\tau^{\wedge} n}^{\prime}: n\langle\omega\rangle\right.$ is a sequence of disjoint open sets in $D$ and $\left\langle C_{\tau^{\wedge} n}: n<\omega\right\rangle$ is a sequence of disjoint open sets in $G$. Thus for each $\varepsilon>0$ there is $n \in \omega$ such that $\mu\left(C_{\tau^{\wedge} n}^{\prime}\right)<\varepsilon$ as well as $\nu\left(C_{\tau^{\frown} n}\right)<\varepsilon$. 
Moreover, for each $m \in \omega$ there is $n \in \omega$ such that $\mu_{V_{m}^{\prime}}\left(V_{m}^{\prime} \cap C_{\tau^{\wedge} n}^{\prime}\right)<\varepsilon$ and $\nu_{V_{m}}\left(V_{m} \cap C_{\tau^{\curvearrowright} n}\right)<\varepsilon$.

By induction, we find a collection of nodes $\tau_{n} \in \omega^{<\omega}$ such that the tree

$$
T=\left\{\tau \in \omega^{<\omega}: \forall n \tau_{n} \nsubseteq \tau\right\}
$$

is such that $\lim (T)$ is nowhere dense, and for each $m<\omega$ we have

$$
\text { either } \quad V_{m}^{\prime} \subseteq \bigcup_{n<\omega} C_{\tau_{n}}^{\prime} \quad \text { or } \quad \mu\left(V_{m} \backslash \bigcup_{n<\omega} C_{\tau_{n}}^{\prime}\right)>0
$$

and

$$
\text { either } \quad V_{m} \subseteq \bigcup_{n<\omega} C_{\tau_{n}} \quad \text { or } \quad \nu\left(V_{m} \backslash \bigcup_{n<\omega} C_{\tau_{n}}\right)>0 .
$$

Along the induction we also construct sequences of reals $\varepsilon_{n} \geq 0$ and $\delta_{n} \geq 0$.

At the $n$-th step of the induction consider the sets $U_{n}^{\prime}=V_{n}^{\prime} \backslash \bigcup_{i<n} C_{\tau_{i}}^{\prime}$ and $U_{n}=$ $V_{n} \backslash \bigcup_{i<n} C_{\tau_{i}}$, which are either empty or of positive measure ( $\mu$ or $\nu$, respectively) by the inductive assumption. Put $\varepsilon_{n}=\mu\left(U_{n}^{\prime}\right), \delta_{n}=\mu\left(U_{n}\right)$. Find $\tau_{n} \in \omega^{<\omega}$ such that $\left.\tau_{n}=\sigma_{n}\right\urcorner k$ for some $k<\omega$ and for each $i \leq n$

- if $\varepsilon_{i}>0$, then $\mu_{V_{i}^{\prime}}\left(C_{\tau_{n}}^{\prime} \cap V_{i}^{\prime}\right)<2^{-n-1} \varepsilon_{i}$,

- if $\delta_{i}>0$, then $\nu_{V_{i}}\left(C_{\tau_{n}} \cap V_{i}\right)<2^{-n-1} \delta_{i}$.

The set

$$
A=G \backslash \bigcup_{n} C_{\tau_{n}}
$$

is of type $\mathbf{G}_{\delta}$. Moreover, it follows from the construction that $\bar{A}=D \backslash \bigcup_{n} C_{\tau_{n}}^{\prime}$ and that $\bar{A}$ is self-supported, so $A \notin \mathcal{E}$ by Lemma 3.3. On the other hand, $A \Vdash \dot{x} \in$ $\lim (T)$, which gives a contradiction, since $\lim (T)$ is nowhere dense.

Corollary 7.2. If $G$ is $\mathbb{P}_{\mathcal{E}}$-generic over $V$, then the extension $V \subseteq V[G]$ is minimal.

Now we will introduce a fusion scheme for the $\sigma$-ideal $\mathcal{E}$. Denote by $G_{\mathcal{E}}$ the following game scheme. In his $n$-th turn, Adam picks $\xi_{n} \in 2^{n}$ such that $\xi_{n} \supsetneq \xi_{n-1}$ $\left(\xi_{-1}=\emptyset\right)$. In her $n$-th turn, Eve picks a basic clopen set $C_{n} \subseteq\left[\xi_{n}\right]$ such that

$$
\mu_{\left[\xi_{n}\right]}\left(C_{n}\right)<\frac{1}{n} .
$$

For a set $A \subseteq 2^{\omega}$ we define the game $G_{\mathcal{E}}(A)$ in the game scheme $G_{\mathcal{E}}$ as follows. Eve wins a play in $G_{\mathcal{E}}(A)$ if

$$
x \notin A \quad \vee \quad \forall^{\infty} n x \in C_{n}
$$

(where $x \in 2^{\omega}$ is the union of the $\xi_{n}$ 's picked by Adam). Otherwise Adam wins.

Proposition 7.3. For any set $A \subseteq 2^{\omega}$, Eve has a winning strategy in $G_{\mathcal{E}}(A)$ if and only if $A \in \mathcal{E}$.

Proof. First suppose that Eve has a winning strategy $S$ in $G_{\mathcal{E}}(A)$. For each $\sigma \in 2^{<\omega}$ consider a partial play $\tau_{\sigma}$ in which Adam picks successively $\sigma\lceil k$ for $k \leq|\sigma|$. Let $C_{\sigma}$ be Eve's next move, according to $S$, after $\tau_{\sigma}$. Put $E_{n}=\bigcup_{\sigma \in 2^{n}} C_{\sigma}$. Clearly $E_{n}$ is a clopen set and $\mu\left(E_{n}\right) \leq 1 / n$. Let $D_{n}=\bigcap_{m \geq n} E_{n}$. Now, each $D_{n}$ is a closed null set and $A \subseteq \bigcup_{n} D_{n}$ since $S$ is a winning strategy. Therefore $A \in \mathcal{E}$.

Conversely, assume that $A \in \mathcal{E}$. There are closed null sets $D_{n}$ such that $A \subseteq$ $\bigcup_{n} D_{n}$. Without loss of generality assume $D_{n} \subseteq D_{n+1}$. Let $T_{n} \subseteq \omega^{<\omega}$ be a tree 
such that $D_{n}=\lim \left(T_{n}\right)$. We define a strategy $S$ for Eve as follows. Suppose Adam has picked $\sigma \in 2^{n}$ in his $n$-th move and consider the tree $T_{n}(\sigma)$. Since $\lim \left(T_{n}(\sigma)\right)$ is of measure zero, there is $k<\omega$ such that

$$
\frac{\left|T_{n}(\sigma) \cap 2^{k}\right|}{2^{k}}<\frac{1}{n} .
$$

Let Eve's answer be the set $\bigcup_{\tau \in T_{n}(\sigma) \cap 2^{k}}[\tau]$. One can readily check that this defines a winning strategy for Eve in $G_{\mathcal{E}}(A)$.

Corollary 7.4. If $B \subseteq 2^{\omega}$ is Borel, then $B \in \mathcal{E}$ if and only if Eve has a winning strategy in $G_{\mathcal{E}}(B)$.

\section{Decomposing Baire Class 1 functions}

Let $X$ and $Y$ be Polish spaces and $f: X \rightarrow Y$ be a Borel function. We say that $f$ is piecewise continuous if $X$ can be covered by a countable family of closed sets on each of which $f$ is continuous.

Recall that a function is $\mathbf{G}_{\delta}$-measurable if preimages of $\mathbf{G}_{\delta}$ sets are $\mathbf{G}_{\delta}$ or, equivalently, if preimages of open sets are $\mathbf{G}_{\delta}$. If $f: X \rightarrow Y$ is a $\mathbf{G}_{\delta}$-measurable function, then preimages of closed sets are $\mathbf{F}_{\sigma}$. Therefore, if $Y$ is zero-dimensional, then preimages of open sets are also $\mathbf{F}_{\sigma}$, so consequently $\boldsymbol{\Delta}_{2}^{0}$.

The following characterization of piecewise continuity has been given by Jayne and Rogers.

Theorem 8.1 (Jayne, Rogers, 7, Theorem 5]). Let $X$ be a Souslin space and $Y$ be a Polish space. A function $f: X \rightarrow Y$ is piecewise continuous if and only if it is $\mathbf{G}_{\delta}$-measurable.

A nice and short proof of the Jayne-Rogers theorem can be found in [13. Classical examples of Borel functions which are not piecewise continuous are the Lebesgue functions $L, L_{1}: 2^{\omega} \rightarrow \mathbb{R}$ (for definitions see [19, Section 1]). For two functions $f: X \rightarrow Y$ and $f^{\prime}: X^{\prime} \rightarrow Y^{\prime}$ we write $f \sqsubseteq f^{\prime}$ if there are topological embeddings $\varphi: X \rightarrow X^{\prime}$ and $\psi: Y \rightarrow Y^{\prime}$ such that $f^{\prime} \circ \varphi=\psi \circ f$. In [19] Solecki strengthened Theorem 8.1 proving the following result.

Theorem 8.2 (Solecki, [19, Theorem 3.1]). Let $X$ be a Souslin space, $Y$ be a Polish space and $f: X \rightarrow Y$ be Baire class 1 . Then

- either $f$ is piecewise continuous

- or $L \sqsubseteq f$, or $L_{1} \sqsubseteq f$.

Theorem 8.1 follows from Theorem 8.2 because neither $L$ nor $L_{1}$ is $\mathbf{G}_{\delta^{-}}$ measurable.

From now until the end of this section we fix a Polish space $X$ and a Baire class 1 , not piecewise continuous function $f: X \rightarrow \omega^{\omega}\left(\omega^{\omega}\right.$ can be replaced with any zero-dimensional Polish space). Consider the $\sigma$-ideal $\mathcal{I}^{f}$ on $X$ generated by closed sets on which $f$ is continuous. We will prove that the forcing $\mathbb{P}_{\mathcal{I}^{f}}$ is equivalent to Miller forcing (see Corollary 8.10).

Suppose $C \subseteq X$ is a compact set and $c: 2^{\omega} \rightarrow C$ is a homeomorphism. We call $(c, C)$ a copy of the Cantor space and denote it by $c: 2^{\omega} \hookrightarrow C \subseteq X$. We denote by $\mathbb{Q}$ the set of all points in $2^{\omega}$ which are eventually equal to 0 . 
Proposition 8.3. Suppose that $G \subseteq X$ is a $\mathbf{G}_{\delta}$ set such that $G \notin \mathcal{I}^{f}$. There exist an open set $U \subseteq \omega^{\omega}$ and a copy of the Cantor space $c: 2^{\omega} \hookrightarrow C \subseteq X$ such that

- $f^{-1}[U] \cap C=c[\mathbb{Q}]$,

- $C \backslash c[\mathbb{Q}] \subseteq G$.

Proof. Denote $\left\{\tau \in 2^{<\omega}: \tau=\emptyset \vee \tau(|\tau|-1)=0\right\}$ by $Q$.

Definition 8.4. A Hurewicz scheme is a Cantor scheme of closed sets $F_{\tau} \subseteq X$ for $\tau \in 2^{<\omega}$ together with a family of points $x_{\tau} \in X$ and clopen sets $U_{\tau} \subseteq \omega^{\omega}$ for $\tau \in Q$ such that

- $x_{\tau \sim 0}=x_{\tau}, U_{\tau \sim 0}=U_{\tau}$,

- $x_{\tau} \in F_{\tau} \cap f^{-1}\left[U_{\tau}\right]$.

Suppose that $G=\bigcap_{n} G_{n}$ with each $G_{n}$ open and $G_{n+1} \subseteq G_{n}$. We will construct a Hurewicz scheme such that for each $\tau \in 2^{n}$ the following two conditions hold:

- $\left(F_{\tau} \backslash f^{-1}\left[\bigcup_{\sigma \in 2^{n} \cap Q} U_{\sigma}\right]\right) \cap G \notin \mathcal{I}^{f}$,

- $F_{\tau-1} \subseteq\left(F_{\tau} \backslash f^{-1}\left[\bigcup_{\sigma \in 2^{n} \cap Q} U_{\sigma}\right]\right) \cap G_{|\tau|}$.

We need the following lemma (its special case can be found in the proof of the Jayne-Rogers theorem in [13]).

Lemma 8.5. If $F$ is a closed set in $X$ and $F \cap G \notin \mathcal{I}^{f}$, then there is $x \in F$ and a clopen set $U \subseteq \omega^{\omega}$ such that $f(x) \in U$ and for each open neighborhood $V$ of $x$

$$
\left(V \backslash f^{-1}[U]\right) \cap G \notin \mathcal{I}^{f} .
$$

Moreover, if $W \subseteq \omega^{\omega}$ is a clopen set such that $F \cap f^{-1}[W] \notin \mathcal{I}^{f}$, then we may require that $U \subseteq W$.

Proof. First let $W=\omega^{\omega}$. Without loss of generality assume that for each nonempty open set $V \subseteq F$ we have $V \cap G \notin \mathcal{I}^{f}$. Suppose that the conclusion is false. We show that $f$ is continuous on $F$, contradicting the fact that $F \cap G \notin \mathcal{I}^{f}$. Pick arbitrary $x \in F$ and a clopen set $U$ such that $f(x) \in U$. By the assumption there is an open neighborhood $V \ni x$ such that $\left(V \backslash f^{-1}[U]\right) \cap G \in \mathcal{I}^{f}$. We claim that $V \subseteq f^{-1}[U]$. Suppose otherwise; then there is $y \in V$ such that $f(y) \notin U$. Pick a clopen set $U^{\prime}$ such that $U^{\prime} \cap U=\emptyset$ and $f(y) \in U^{\prime}$. Again, by the assumption there is an open neighborhood $V^{\prime}$ of $y$ such that $\left(V^{\prime} \backslash f^{-1}\left[U^{\prime}\right]\right) \cap G \in \mathcal{I}^{f}$. Now $V^{\prime \prime}=V \cap V^{\prime}$ is a nonempty open set and since $U^{\prime} \cap U=\emptyset$ we have that

$$
V^{\prime \prime} \cap G \subseteq\left(V \backslash f^{-1}[U]\right) \cap G \cup\left(V^{\prime} \backslash f^{-1}\left[U^{\prime}\right]\right) \cap G .
$$

This shows that $V^{\prime \prime} \cap G \in \mathcal{I}^{f}$, a contradiction.

Now, if $W \subseteq \omega^{\omega}$ is a clopen set such that $F \cap f^{-1}[W] \notin \mathcal{I}^{f}$, then $f^{-1}[W] \cap F$ is an $\mathbf{F}_{\sigma}$ set since $f$ is Baire class 1. So there is a closed set $F^{\prime} \subseteq F$ such that $F^{\prime} \notin \mathcal{I}^{f}$ and $f\left[F^{\prime}\right] \subseteq W$. Applying the previous argument to $F^{\prime}$ we get a clopen set $U$ such that $U \subseteq W$. This ends the proof.

Now we construct a Hurewicz scheme. First use Lemma 8.5 to find $x_{\emptyset}, U_{\emptyset}$ and put $F_{\emptyset}=X$. Suppose the scheme is constructed up to the level $n-1$.

First we will construct $U_{\sigma \frown 0}$ and $x_{\sigma \frown 0}$ for each $\sigma \in 2^{n-1} \backslash Q$ (recall that for $\sigma \in 2^{n-1} \cap Q$ we put $U_{\sigma \frown 0}=U_{\sigma}$ and $x_{\sigma \frown 0}=x_{\sigma}$ ).

For each $\sigma \in 2^{n-1} \backslash Q$ find a nonempty, perfect closed set $C_{\sigma} \subseteq \omega^{\omega}$ such that $F_{\sigma} \cap f^{-1}[V] \notin \mathcal{I}^{f}$ for each nonempty relatively clopen set $V \subseteq C_{\sigma}$ (this is done by removing from $\omega^{\omega}$ those clopen sets $U$ such that $\left.F_{\tau} \cap f^{-1}[U] \in \mathcal{I}^{f}\right)$. 
Lemma 8.6. There is a sequence of nonempty clopen (in $\left.\omega^{\omega}\right)$ sets $\left\langle W_{\tau}: \tau \in\right.$ $\left.2^{n-1} \backslash Q\right\rangle$ such that

- $W_{\tau} \cap C_{\tau} \neq \emptyset$,

- for each $\sigma \in 2^{n-1} \cap Q$ and for each open neighborhood $V$ of $x_{\sigma}$ we have

$$
\left(\left(V \backslash \bigcup_{\sigma^{\prime} \in 2^{n-1} \cap Q} f^{-1}\left[U_{\sigma^{\prime}}\right]\right) \backslash \bigcup_{\tau \in 2^{n-1} \backslash Q} f^{-1}\left[W_{\tau}\right]\right) \cap G \notin \mathcal{I}^{f} .
$$

Proof. Enumerate $2^{n-1} \backslash Q$ in a sequence $\left\langle\tau_{i}: i<2^{n-2}\right\rangle$ and construct the sets $W_{\tau_{i}}$ by induction on $i<2^{n-2}$. Fix $i<2^{n-2}$ and suppose that $W_{\tau_{j}}$ are already defined for $j<i$ and

$$
\left(\left(V \backslash \bigcup_{\sigma \in 2^{n-1} \cap Q} f^{-1}\left[U_{\sigma}\right]\right) \backslash \bigcup_{j<i} f^{-1}\left[W_{\tau_{j}}\right]\right) \cap G \notin \mathcal{I}^{f} .
$$

Claim. If $O_{0}$ and $O_{1}$ are two disjoint nonempty clopen sets in $\omega^{\omega}$, then for each $\sigma \in 2^{n-1} \cap Q$ there exists $k \in\{0,1\}$ such that for each open neighborhood $V$ of $x_{\sigma}$ the following holds:

$$
\left(\left(V \backslash \bigcup_{\sigma \in 2^{n-1} \cap Q} f^{-1}\left[U_{\sigma}\right]\right) \backslash \bigcup_{j<i} f^{-1}\left[W_{\tau_{j}}\right]\right) \cap G \backslash f^{-1}\left[O_{k}\right] \notin \mathcal{I}^{f} .
$$

Proof. Notice that for a single open neighborhood $V$ of $x_{\sigma}$ one $k \in\{0,1\}$ is good. If $\left\langle V_{n}: n<\omega\right\rangle$ is a base at $x_{\sigma}$, then some $k \in\{0,1\}$ is good for infinitely many of them.

Enumerate $2^{n-1} \cap Q$ in a sequence $\left\langle\sigma_{k}: k<2^{n-2}\right\rangle$. Using the above Claim and the fact that $C_{\tau_{i}}$ is perfect, find a decreasing sequence of nonempty clopen sets $O_{k} \subseteq \omega^{\omega}$ for $k<2^{n-2}$ such that

- $O_{k} \subseteq O_{k-1}$,

- $O_{k} \cap C_{\tau_{i}} \neq \emptyset$,

- for each open neighborhood $V$ of $x_{\sigma_{k}}$

$$
\left(\left(V \backslash \bigcup_{\sigma \in 2^{n-1} \cap Q} f^{-1}\left[U_{\sigma}\right]\right) \backslash \bigcup_{j<i} f^{-1}\left[W_{\tau_{j}}\right]\right) \cap G \backslash f^{-1}\left[O_{k}\right] \notin \mathcal{I}^{f} .
$$

Finally, let $W_{\tau_{i}}$ be the last of the $O_{k}$ 's.

By the assumption on $C_{\tau}$ 's, we have $F_{\tau} \cap f^{-1}\left[W_{\tau}\right] \notin \mathcal{I}^{f}$, for each $\tau \in 2^{n-1} \backslash Q$. Using Lemma 8.5. for each $\tau \in 2^{n-1} \backslash Q$ find a clopen set $U_{\tau^{\wedge} 0} \subseteq W_{\tau}$ and a point $x_{\tau \sim 0}$ such that the assertion of Lemma 8.5 holds.

Now all $U_{\tau}$ and $x_{\tau}$ for $\tau \in 2^{n} \cap Q$ are defined and we need to find sets $F_{\sigma}$ for $\sigma \in 2^{n}$.

Claim. For each $\sigma \in 2^{n-1}$ there are two disjoint $\mathcal{I}^{f}$-positive closed sets $F_{\sigma \frown 0}, F_{\sigma \wedge 1} \subseteq$ $F_{\sigma}$ of diameters less than $1 / n$ such that

$$
F_{\sigma \frown 1} \subseteq\left(F_{\sigma} \backslash \bigcup_{\tau \in 2^{n} \cap Q} f^{-1}\left[U_{\tau}\right]\right) \cap G_{n-1}
$$

and $F_{\sigma \frown 0}$ contains $x_{\sigma \frown 0}$. 
Proof. For each $\sigma \in 2^{n-1}$ take an open neighborhood $V_{\sigma}$ of $x_{\sigma \frown 0}$ of diameter $<1 / n$. The set

$$
V_{\sigma} \backslash f^{-1}\left[\bigcup_{\tau \in 2^{n} \cap Q} U_{\tau}\right]
$$

is $\mathbf{F}_{\sigma}$ (since $f$ is Baire class 1 ) which has $\mathcal{I}^{f}$-positive intersection with $G$. Thus it has a closed subset $F$ such that $F$ also has $\mathcal{I}^{f}$-positive intersection with $G$. Now, the set $F \cap G_{n-1}$ is $\mathbf{F}_{\sigma}$, so find $F_{\sigma \frown 1}$ which is a closed subset of $F \cap G_{n-1}$ and has $\mathcal{I}^{f}$-positive intersection with $F \cap G$. Let $F_{\sigma \frown 0}$ be a closed neighborhood of $x_{\sigma \frown 0}$, disjoint from $F_{\sigma \frown 1}$.

This ends the construction of the Hurewicz scheme. To finish the proof, we put $U=\bigcup_{\tau \in 2^{<\omega}} U_{\tau}, C=\bigcap_{n<\omega} \bigcup_{\tau \in 2^{n}} F_{\tau}$ and $c: 2^{\omega} \hookrightarrow C \subseteq X$ such that $c(x) \in$ $\bigcap_{n<\omega} F_{x\lceil n}$ for each $x \in 2^{\omega}$.

Proposition 8.7. The $\sigma$-ideal $\mathcal{I}^{f}$ is $\boldsymbol{\Pi}_{1}^{1}$ on $\boldsymbol{\Sigma}_{1}^{1}$.

Proof. This follows from Corollary 3.8 since the family of closed sets on which $f$ is continuous is hereditary and $\boldsymbol{\Pi}_{1}^{1}$.

Remark 8.8. Using Proposition 8.3 we can explicitly write the formula defining the set of closed sets in $\mathcal{I}^{f}$. Let $\tilde{K} \subseteq \omega^{\omega} \times X$ be the universal closed set. Notice that $\tilde{K}_{x} \notin \mathcal{I}^{f}$ if and only if

$$
\begin{gathered}
\exists U \subseteq 2^{\omega} \text { open } \exists c: 2^{\omega} \rightarrow \tilde{K}_{x} \text { topological embedding } \\
\left(c\left[2^{\omega}\right] \cap f^{-1}[U] \text { is dense in } c\left[2^{\omega}\right]\right) \wedge\left(c\left[2^{\omega}\right] \backslash f^{-1}[U] \text { is dense in } c\left[2^{\omega}\right]\right) .
\end{gathered}
$$

Indeed, the left-to-right implication follows from Proposition 8.3 (when $G=X$ ). The right-to-left implication holds because the set $f^{-1}[U]$ is an $\mathbf{F}_{\sigma}$ set which is dense and meager on $c\left[2^{\omega}\right]$, therefore it cannot be a relative $\mathbf{G}_{\delta}$ set on $c\left[2^{\omega}\right]$. Hence, by the Jayne-Rogers theorem we have that $f$ is not piecewise continuous on $c\left[2^{\omega}\right]$ and $\tilde{K}_{x} \notin \mathcal{I}^{f}$.

Now, the above formula is $\boldsymbol{\Sigma}_{1}^{1}$. Indeed, it is routine to write a $\boldsymbol{\Sigma}_{1}^{1}$ formula saying that $c: 2^{\omega} \rightarrow K_{x}$ is a topological embedding. The first clause of the conjunction can be written as

$$
\forall \tau \in 2^{<\omega} \exists x \in[\tau] \quad f(c(x)) \in U,
$$

which is $\Sigma_{1}^{1}$, and analogously we can rewrite the second clause.

If $G \subseteq X$ is a $\mathbf{G}_{\delta}$ set and $b: \omega^{\omega} \rightarrow G$ is a homeomorphism, then we call $(b, G) a$ copy of the Baire space and denote it by $b: \omega^{\omega} \hookrightarrow G \subseteq X$.

Proposition 8.9. For any $B \in \mathbb{P}_{\mathcal{I}^{f}}$ there is an $\mathcal{I}^{f}$-positive $\mathbf{G}_{\delta}$ set $G \subseteq B$ and a copy of the Baire space $b: \omega^{\omega} \hookrightarrow G \subseteq X$ such that

$$
\mathcal{I}^{f}\left\lceil G=\left\{b[A]: A \subseteq \omega^{\omega}, A \in \mathbf{K}_{\sigma}\right\} .\right.
$$

Proof. By Solecki's theorem and the continuous reading of names we may assume that $B$ is of type $\mathbf{G}_{\delta}$ and $f$ is continuous on $B$. Applying Proposition 8.3 we get a copy of the Cantor space $c: 2^{\omega} \hookrightarrow C \subseteq X$ and an open set $U \subseteq \omega^{\omega}$ such that $f^{-1}[U] \cap C=c[\mathbb{Q}]$ and $C \backslash c[\mathbb{Q}] \subseteq B$. Let $G=C \backslash c[\mathbb{Q}]$. Via a natural homeomorphism of $\omega^{\omega}$ and $2^{\omega} \backslash \mathbb{Q}$ we get a copy of the Baire space $b: \omega^{\omega} \hookrightarrow G \subseteq X$. Note that $C \notin \mathcal{I}^{f}$ (by Theorem $\left[8.1\right.$, since $f^{-1}[U] \cap C$ is not $\mathbf{G}_{\delta}$ in $C$ ) and hence also $G \notin \mathcal{I}^{f}$. 
The $\sigma$-ideal $\mathcal{I}^{f}\lceil G$ is generated by the sets $D \cap G$ for $D \subseteq C$ closed such that $f\left\lceil D\right.$ is continuous. The $\sigma$-ideal $\left\{b[A]: A \subseteq \omega^{\omega}, A \in \mathbf{K}_{\sigma}\right\}$ is generated by compact subsets of $G$. We need to prove that these two families generate the same $\sigma$-ideals on $G$.

If $D \subseteq G$ is compact, then $D$ is closed in $C$ and $f$ is continuous on $D$ because $f$ is continuous on $G$. Hence $D=D \cap G \in \mathcal{I}^{f}\lceil G$.

If $D \subseteq C$ is such that $f$ is continuous on $D$, then $D \cap G=\left(f\lceil D)^{-1}\left[\omega^{\omega} \backslash U\right]\right.$ is a closed in $D$ subset of $G$, therefore compact.

This ends the proof.

As an immediate consequence of Proposition 8.9 we get the following corollary.

Corollary 8.10. The forcing $\mathbb{P}_{\mathcal{I}^{f}}$ is equivalent to Miller forcing.

Recall that Miller forcing has the following property: if $x \in \omega^{\omega}$ is a real added in a generic extension, then either $x$ is a ground model real or there is a ground model Borel automorphism $\varphi: \omega^{\omega} \rightarrow \omega^{\omega}$ such that $\varphi(x)$ is the generic real. From this and the above corollary we get the following result, which does not mention forcing at all.

Corollary 8.11. For any $\mathcal{I}^{f}$-positive Borel set $B$ and any Borel function $g: B \rightarrow$ $\omega^{\omega}$ there is an $\mathcal{I}^{f}$-positive set $C \subseteq B$ such that $g\lceil C$ is either 1 -1 or constant.

Recall a theorem of Kechris, Louveau and Woodin [10, Theorem 7] which says that any coanalytic $\sigma$-ideal of compact sets in a Polish space is either a $\mathbf{G}_{\delta}$ set or is $\Pi_{1}^{1}$-complete. If $X$ is compact, then $\mathcal{I}^{f} \cap K(X)$ is a coanalytic $\sigma$-ideal of compact sets by Proposition 8.7 .

Proposition 8.12. $\mathcal{I}^{f} \cap F(X)$ is a $\Pi_{1}^{1}$-complete set in $F(X)$.

Proof. As in Proposition 8.9 take $c: 2^{\omega} \hookrightarrow C \subseteq X$ as a copy of the Cantor space and $b: \omega^{\omega} \hookrightarrow G \subseteq X$ a copy of the Baire space, and $G \subseteq C$ a dense $\mathbf{G}_{\delta}$ set in $C$. Recall that the Borel structure on $F(C)$ is induced from the topology of the hyperspace.

It is well known (see [9, Exercise 27.9]) that the set $\mathbf{K}_{\sigma} \cap F\left(\omega^{\omega}\right)$ is a $\boldsymbol{\Pi}_{1}^{1}$-complete set in $F\left(\omega^{\omega}\right)$. Let $\varphi: F\left(\omega^{\omega}\right) \rightarrow K(C)$ be the function

$$
F\left(\omega^{\omega}\right) \ni F \mapsto \overline{b[F]} \in K(C),
$$

where $\bar{A}$ denotes the closure of $A$ in $C$ (for $A \subseteq G$ ). It is routine to check that $\varphi$ is Borel-measurable. By Proposition 8.9 we have $\varphi^{-1}\left[\mathcal{I}^{f}\right]=\mathbf{K}_{\sigma}$. This proves that $\mathcal{I}^{f}$ is $\boldsymbol{\Pi}_{1}^{1}$-complete.

Piecewise continuity of functions from $\omega^{\omega}$ to $\omega^{\omega}$ has already been investigated from the game-theoretic point of view. In 20] Van Wesep introduced the Backtrack Game $G_{\mathrm{B}}(g)$ for functions $g: \omega^{\omega} \rightarrow \omega^{\omega}$. Andretta [1, Theorem 21] characterized piecewise continuity of a function $g$ in terms of the existence of a winning strategy for one of the players in the game $G_{\mathrm{B}}(g)$.

For a Borel not piecewise continuous function $g: \omega^{\omega} \rightarrow \omega^{\omega}$, the Backtrack Game can be used to define a fusion scheme for the $\sigma$-ideal $\mathcal{I}^{g}$. In the remaining part of this section, we will show a very natural fusion scheme for $\mathcal{I}_{g}$ when $g: 2^{\omega} \rightarrow 2^{\omega}$ is Borel, not piecewise continuous.

Recall that partial continuous functions from $2^{\omega}$ to $2^{\omega}$ with closed domains can be coded by monotone functions from $2^{<\omega}$ into $2^{<\omega}$ (see [9, Section $\left.2 \mathrm{~B}\right]$ ). 
If $T \subseteq 2^{<\omega}$ is a finite tree, $m: T \rightarrow 2^{<\omega}$ is a monotone function and $n<\omega$, then we say that $(T, m)$ is a monotone function of height $n$ if $m(\tau)$ is of length $n$ for each terminal node $\tau$ of $T$. We say that $\left(T_{1}, m_{1}\right)$ extends $\left(T_{0}, m_{0}\right)$ if $T_{1}$ is an end-extension of $T_{0}$ and $m_{1} \supseteq m_{0}$.

We define the game scheme $G_{\mathrm{pc}}$ as follows. In his $n$-th move, Adam picks $\xi_{n} \in \omega^{n}$ such that $\xi_{n} \supseteq \xi_{n-1}\left(\xi_{-1}=\emptyset\right)$. In her $n$-th turn, Eve constructs a sequence of finite monotone functions $\left\langle H_{i}^{n}: i<\omega\right\rangle$ such that

- $\forall^{\infty} i H_{i}^{n}=\emptyset$,

- $H_{i}^{n}$ extends $H_{i}^{n-1}$,

- $H_{i}^{n}$ is a monotone function of height $n$ whenever $H_{i}^{n} \neq \emptyset$.

In each play in $G_{\mathrm{pc}}$, for each $i<\omega$ we have that $H_{i}=\bigcup_{n<\omega} H_{i}^{n}$ is a monotone function which defines a partial continuous function $h_{i}$ with closed domain (possibly empty).

Let $g: \omega^{\omega} \rightarrow \omega^{\omega}$ be a not piecewise continuous function and $B \subseteq \omega^{\omega}$. The game $G_{\mathrm{pc}}^{g}(B)$ is a game in the game scheme $G_{\mathrm{pc}}$ with the following payoff set. Eve wins a play $p$ in $G_{\mathrm{pc}}^{g}(B)$ if for $x=\bigcup_{n<\omega} \xi_{n}\left(\xi_{n}\right.$ is the $n$-th move of Adam in $p$ )

$$
x \notin B \quad \vee \quad \exists i \in \omega\left(x \in \operatorname{dom}\left(h_{i}\right) \wedge g(x)=h_{i}(x)\right)
$$

(where the functions $h_{i}$ are computed from Eve's moves as above). Otherwise Adam wins $p$.

Proposition 8.13. For any set $A \subseteq \omega^{\omega}$, Eve has a winning strategy in the game $G_{\mathrm{pc}}^{g}(A)$ if and only if $A \in \mathcal{I}^{g}$.

Proof. If $A \in \mathcal{I}^{g}$, then there are closed sets $C_{n} \subseteq 2^{\omega}$ such that $A \subseteq \bigcup_{n} C_{n}$ and $g\left\lceil C_{n}\right.$ is continuous. Each function $g\left\lceil C_{n}\right.$ has its monotone function $G_{n}$ and Eve's strategy is simply to rewrite the $G_{n}$ 's.

On the other hand, suppose that there is a winning strategy for Eve and let $S$ be the tree of this strategy. The nodes of $S$ are determined by Adam's moves, so $S$ is isomorphic to $2^{<\omega}$. For $\tau \in T$ let $m_{k}^{\tau}: T_{k}^{\tau} \rightarrow 2^{<\omega}$ be the monotone function $H_{k}$ defined by Eve in her last move of the partial play $\tau$. Denote by $H_{k}^{\tau}[\tau]$ the restriction of $m_{k}^{\tau}$ to $T_{k}^{\tau}(\tau)$. Put $G_{k}=\bigcup_{\tau \in T} H_{k}^{\tau}[\tau]$ and let $g_{k}$ be the partial continuous function with closed domain determined by the monotone function $G_{k}$. It follows from the fact that $S$ is winning for Eve, that $g\left\lceil A \subseteq \bigcup_{n} g_{n}\right.$. This proves that $A \in \mathcal{I}^{g}$.

Corollary 8.14. If $B \subseteq 2^{\omega}$ is Borel and $g: 2^{\omega} \rightarrow 2^{\omega}$ is a Borel, not piecewise continuous function, then $B \in \mathcal{I}^{g}$ if and only if Eve has a winning strategy in $G_{\mathrm{pc}}^{g}(B)$.

\section{ACKNOWLEDGEMENTS}

The author would like to thank Janusz Pawlikowski for all the helpful remarks and stimulating discussions. He would also like to thank Stevo Todorčević, Sy Friedman, Tamás Mátrai and Jindřich Zapletal for many useful comments.

\section{REFERENCES}

[1] Andretta A., More on Wadge determinacy, Annals of Pure and Applied Logic, Vol. 144 (2006), 2-32. MR 2279651 (2008k:03094)

[2] Bartoszyński T., Judah H., Set Theory. On the Structure of the Real Line, A K Peters, 1995. MR:1350295 (96k:03002) 
[3] Bartoszyński T., Shelah S., Closed measure zero sets, Annals of Pure and Applied Logic, Vol. 58 (1992), 93-110. MR.1186905 (94b:03084)

[4] Ciesielski K., Pawlikowski J., The Covering Property Axiom, CPA: A Combinatorial Core of the Iterated Perfect Set Model, Cambridge University Press, 2004. MR.2176267 (2006f:03002)

[5] Farah I., Zapletal J., Four and more, Annals of Pure and Applied Logic, Vol. 140 (2006), 3-39. MR2224046 (2007a:03063)

[6] Ishiu T., $\alpha$-properness and Axiom A, Fundamenta Mathematicae, Vol. 186 (2005), 25-37. MR2163100 (2006g:03081)

[7] Jayne J. E., Rogers C. A., First level Borel functions and isomorphism, Journal de Mathématiques Pures et Appliquées, Vol. 61 (1982), 177-205. MR673304 (84a:54072)

[8] Kanovei V., Non-Glimm-Effros equivalence relations at second projective level, Fundamenta Mathematicae Vol. 154 (1997), 1-35. MR1472849 (99j:03040)

[9] Kechris A., Classical Descriptive Set Theory, Graduate Texts in Mathematics 156, SpringerVerlag, 1995. MR.1321597 (96e:03057)

[10] Kechris A. S., Louveau A., Woodin W. H., The structure of $\sigma$-ideals of compact sets, Transactions of the American Mathematical Society, Vol. 301 (1987), 263-288. MR.879573(88f:03042)

[11] Mátrai T., Infinite dimensional perfect set theorems, preprint.

[12] Moschovakis Y., Descriptive set theory, Studies in Logic and the Foundations of Mathematics, Vol. 100, North-Holland Publishing Company, Amsterdam, 1980. MR.561709 (82e:03002)

[13] Motto-Ros L., Semmes B., A new proof of the Jayne-Rogers Theorem, Real Analysis Exchange, Vol. 35, No. 1(2010), 195-203. MR2657295

[14] Mycielski J., Some new ideals of sets on the real line, Colloquium Mathematicum, Vol. 20 (1969), 71-76. MR0241595 (39:2934)

[15] Pawlikowski J., Definable forcing, unpublished lecture notes.

[16] Petruska G., On Borel sets with small covers, Real Analysis Exchange, Vol. 18 (1992-93), 330-338. MR1228398 (95g:28003a)

[17] Schmidt W. M., On badly approximable numbers and certain games, Transactions of the American Mathematical Society, Vol. 123 (1966), 178-199. MR0195595 (33:3793)

[18] Solecki S., Covering analytic sets by families of closed sets, Journal of Symbolic Logic, Vol. 59, No. 3 (1994), 1022-1031. MR 1295987 (95g:54033)

[19] Solecki S., Decomposing Borel sets and functions and the structure of Baire class 1 functions, Journal of the American Mathematical Society, Vol. 11, No. 3 (1998), 521-550. MR.1606843 (99h:26010)

[20] Van Wesep R., Subsystems of second-order arithmetic and descriptive set theory under the Axiom of Determinateness, Ph.D. Thesis, University of California, Berkeley, 1977. MR2627617

[21] Zapletal J., Descriptive Set Theory and Definable Forcing, Memoirs of the American Mathematical Society, 2004. MR2023448 (2005e:03107)

[22] Zapletal J., Forcing Idealized, Cambridge Tracts in Mathematics 174, 2008. MR.2391923 (2009b:03002)

[23] Zapletal J., Forcing with ideals of closed sets, Commentationes Mathematicae Universitatis Carolinae, Vol. 43, No. 1 (2002), 181-188. MR.1903318 (2003c:03092)

Mathematical Institute, WrocŁaw University, Pl. Grunwaldzki 2/4, 50-384 WrocŁaW, POLAND

E-mail address: sabok@math.uni.wroc.pl 